\title{
Adapting to life: ocean biogeochemical modelling and adaptive remeshing
}

\author{
J. Hill ${ }^{1}$, E. E. Popova ${ }^{2}$, D. A. Ham ${ }^{3}$, M. D. Piggott ${ }^{1,4}$, and M. Srokosz ${ }^{2}$ \\ ${ }^{1}$ Applied Modelling and Computation Group, Department of Earth Science and Engineering, Imperial College London, \\ SW7 2AZ, UK \\ ${ }^{2}$ National Oceanography Centre, Southampton, University of Southampton Waterfront Campus, European Way, \\ Southampton, SO14 3ZH, UK \\ ${ }^{3}$ Applied Mathematics and Mathematical Physics, Department of Mathematics, Imperial College London, SW7 2AZ, UK \\ ${ }^{4}$ Grantham Institute for Climate Change, Imperial College London, SW7 2AZ, UK
}

Correspondence to: J. Hill (jon.hill@imperial.ac.uk)

Received: 23 September 2013 - Published in Ocean Sci. Discuss.: 5 November 2013

Revised: 30 March 2014 - Accepted: 1 April 2014 - Published: 9 May 2014

\begin{abstract}
An outstanding problem in biogeochemical modelling of the ocean is that many of the key processes occur intermittently at small scales, such as the sub-mesoscale, that are not well represented in global ocean models. This is partly due to their failure to resolve sub-mesoscale phenomena, which play a significant role in vertical nutrient supply. Simply increasing the resolution of the models may be an inefficient computational solution to this problem. An approach based on recent advances in adaptive mesh computational techniques may offer an alternative. Here the first steps in such an approach are described, using the example of a simple vertical column (quasi-1-D) ocean biogeochemical model.

We present a novel method of simulating ocean biogeochemical behaviour on a vertically adaptive computational mesh, where the mesh changes in response to the biogeochemical and physical state of the system throughout the simulation. We show that the model reproduces the general physical and biological behaviour at three ocean stations (India, Papa and Bermuda) as compared to a high-resolution fixed mesh simulation and to observations. The use of an adaptive mesh does not increase the computational error, but reduces the number of mesh elements by a factor of $2-3$. Unlike previous work the adaptivity metric used is flexible and we show that capturing the physical behaviour of the model is paramount to achieving a reasonable solution. Adding biological quantities to the adaptivity metric further refines the solution. We then show the potential of this method in two
\end{abstract}

case studies where we change the adaptivity metric used to determine the varying mesh sizes in order to capture the dynamics of chlorophyll at Bermuda and sinking detritus at Papa. We therefore demonstrate that adaptive meshes may provide a suitable numerical technique for simulating seasonal or transient biogeochemical behaviour at high vertical resolution whilst minimising the number of elements in the mesh. More work is required to move this to fully 3-D simulations.

\section{Introduction}

Biogeochemical processes in the ocean are a key component of Earth's climate system and, in particular, of the carbon cycle. Understanding of the latter is crucial for projections of future climate change. However, modelling the biogeochemistry of the oceans presents a formidable challenge as many of the key processes occur at scales (both horizontal and vertical) not resolved by state-of-the-art climate models. Both mesoscale and sub-mesoscale processes are thought to be important - for example, in the supply of nutrients to the surface water of the oligotrophic gyres that cover one-third of the earth's surface (Oschlies, 2002; McGillicuddy et al., 2003; Lévy et al., 2012). It is generally considered that the representation of the physical processes is a key part in correctly representing ocean biogeochemistry (e.g. Friedrichs et al., 2006), including the representation of the light spectra 
(Kettle and Merchant, 2008). Furthermore, many of the processes affecting biogeochemistry at the mesoscale and submesoscale have significant vertical structure (Lévy et al., 2012), meaning that vertical resolution is also important. In addition, the surface fluxes that drive mixed-layer depth (MLD) behaviour can greatly affect the vertical nutrient fluxes (Berline et al., 2007), highlighting the importance of the representation of vertical processes. Thus there is a need to have sufficiently high vertical resolution to correctly represent vertical advection together with mixed-layer deepening and shallowing. Current ocean models use decreasing resolution with increasing depth, concentrating resolution in the upper layers (e.g. Popova et al., 2006). Multiscale resolution is possible using an unstructured mesh where horizontal resolution can vary spatially by orders of magnitude, but the same method can be applied in the vertical also. A number of coastal and regional models use such an approach to model complex coastlines and bathymetries (e.g. Ji et al., 2008; Luo et al., 2012). These models have been successfully coupled to biogeochemical models to study nutrient cycling and plankton blooms (Khangaonkar et al., 2012; Ji et al., 2008), and water quality (Menendez et al., 2013). In addition to multiscale resolution which alters resolution spatially, it is also possible to alter the resolution temporally - mesh adaptivity, which aims to alter resolution only when and where it is required (e.g. Hiester et al., 2011). This approach aims to reduce the number of elements required whilst maintaining some measure of error. Here, as a first step, the suitability of mesh adaptivity for providing appropriate vertical resolution is tested using a simple vertical-column coupled physics and ecosystem model. We neglect vertical advection terms and focus on mixed-layer dynamics only.

The behaviour of ocean ecosystems, and the associated biogeochemistry, is driven largely by physical processes (stirring and mixing). These vary depending on location; for example, differing between the subpolar and subtropical gyres. Therefore, simulations at different locations in the ocean may require different resolution structure (meshes) in the vertical. Adaptivity should allow the best mesh structure to be chosen for each location. By carefully selecting the adaptivity metric and parameters controlling the mesh, computational cost can in principle be minimised by reducing the number of degrees of freedom (Hiester et al., 2011; Hill et al., 2012). There is also a need to conserve biogeochemical quantities, so interpolation between meshes during adaptation can therefore be key in ensuring conservation. Adaptivity has been used previously in ocean-type settings. Hill et al. (2012) showed that adaptivity can reduce the number of elements required to model the mixed layer, using Fluidity, the model also used here. Adaptive techniques have been shown to reduce levels of numerical mixing in a number of idealised examples (Hofmeister et al., 2010). Other models have shown effective use of adaptive grids to improve the representation of vertical mixing processes (Burchard and Beckers, 2004). This paper represents the first study to assess the effect of adaptive meshes on ocean ecosystem model numerical accuracy.

The adaptive mesh technique used in Fluidity differs from previous implementations of adaptive mesh techniques used in similar models in that the number of elements (or in the case of finite-difference models, grid points) can change throughout the simulation. For example, in both Burchard and Beckers (2004) and Hanert et al. (2006) the number of grid points remains fixed: the adaptive mesh moves them to locations to minimise an error metric; in essence a mesh movement algorithm. The techniques of Burchard and Beckers (2004) have been extended to 3-D by allowing each horizontal location to have a different vertical mesh (Hofmeister et al., 2010). Again, the number of grid points is fixed. In addition to the number of elements being able to vary throughout the simulation, the model presented here also allows the adaptivity metric weights to be user defined, giving a great deal of flexibility on the adaptivity metric composition. This allows a comparison of using only biological tracers in the adaptivity metric, only physical variables, or a combination of both.

In order to examine a wide range of ocean conditions, three ocean stations (Fig. 1) were chosen to test the performance of mesh adaptivity in conjunction with ocean biogeochemistry models. These were Ocean Weather Station Papa, Ocean Weather Station India and the Bermuda Atlantic Time-series. These stations show very different mixed-layer and biological behaviours and so test a model's ability to accurately simulate a range of physical and biological behaviours. Whilst Papa is ideal for carrying out 1-D studies due to the lack of significant horizontal advection (Denman and Miyake, 1973; Gaspar et al., 1990; Burchard and Bolding, 2001), India and Bermuda both experience significant horizontal advection. Previous attempts to model Bermuda in one dimension have resorted to ad hoc "fixes" (Anderson and Pondaven, 2003; Weber et al., 2007) in order to simulate the physical and biological behaviour here. However, the aims of these previous studies were to understand the processes occurring in more detail. In this study we are concerned with how well adaptive remeshing can replicate the results of a fixed-mesh simulation whilst minimising the number of elements used. We therefore do not expect a perfect match to observed data for these two stations, but the simulations must replicate the general observed behaviour at all three stations. In addition, we do not necessarily expect model skill to increase when using adaptive remeshing. We are instead examining the numerical response of the model. Without numerical confidence in the model, altering biological parameters to tune the model may "lead to misconceptions in the interpretation of ecosystem dynamics" (Oschlies and Garçon, 1999).

In Sect. 2 the numerical model used in this study is described, including the biogeochemical model used, turbulence parameterisation and mesh adaptivity algorithm. This model is then evaluated in Sect. 3, before the results from fixed-mesh simulations are described. Section 4 then presents 


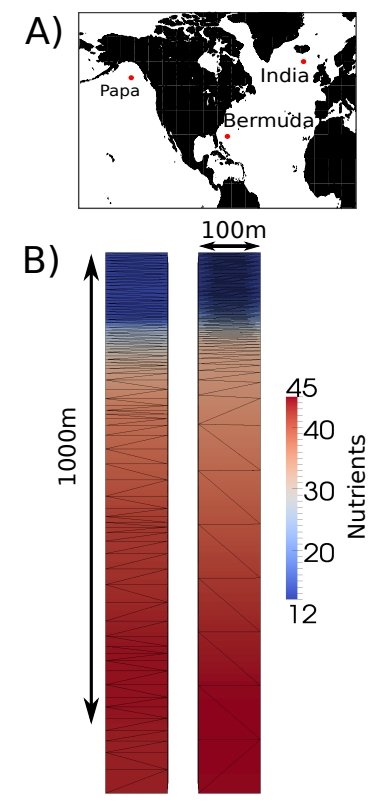

Fig. 1. Map of station locations (A) and 2-D view of the model domain at showing two different meshes produced by the adaptivity algorithm (B).

results from the adaptive mesh simulation. Finally, two experiments are described in which the mesh is adapted to concentrate resolution not only in critical regions such as at the MLD, but also to track sinking detritus at Station Papa to well below the mixed layer depth and the subsurface chlorophyll maximum at Bermuda, which occurs below the mixed layer. The paper then assesses the merits of the adaptive algorithm presented and draws some conclusions.

\section{Methods}

Here, the non-hydrostatic Boussinesq equation system is considered in the context of Fluidity (Ford et al., 2004; Pain et al., 2005; Piggott et al., 2008b), a highly flexible finite element/control volume modelling framework which allows for the numerical solution of the following set of equations:

$$
\begin{aligned}
& \frac{\partial \boldsymbol{u}}{\partial t}+\boldsymbol{u} \cdot \nabla \boldsymbol{u}+f \underline{k} \times \boldsymbol{u}= \\
& -\nabla\left(\frac{p}{\rho_{0}}\right)-\frac{\rho}{\rho_{0}} g \underline{k}+\nabla \cdot(\boldsymbol{v} \nabla \boldsymbol{u}), \\
& \nabla \cdot \boldsymbol{u}=0, \\
& \frac{\partial T}{\partial t}+\boldsymbol{u} \cdot \nabla T=\nabla \cdot\left(\kappa_{T} \nabla T\right), \\
& \frac{\partial S}{\partial t}+\boldsymbol{u} \cdot \nabla S=\nabla \cdot\left(\kappa_{S} \nabla S\right), \\
& \rho \equiv \rho(T, S),
\end{aligned}
$$

where $\boldsymbol{u}$ is the 3-D velocity vector, $t$ represents time, $p$ is the pressure, $g$ is the acceleration due to gravity acting in the
$\underline{k}=(0,0,1)^{T}$ direction, $T$ is temperature and $S$ is salinity. $\rho$ is the density which is given in terms of an equation of state function with temperature and salinity as input arguments, and $\rho_{0}$ is a constant background value for density. $\boldsymbol{v}$ is the tensor of kinematic viscosities and $\kappa_{T}, \kappa_{S}$ are the thermal and saline diffusivity tensors respectively. $f$ is the Coriolis parameter which in this work is assumed constant. We also assume, for simplicity, a Cartesian coordinate system with $\underline{k}$ pointing in the direction of gravity.

The above equations were discretised on an unstructured mesh of tetrahedral elements using the finite-element method. The form of the discretisation is determined by the order of the polynomials used for the different solution variables and whether or not they are continuous or discontinuous across element faces. A constant time step of $360 \mathrm{~s}$ is used with Crank-Nicolson temporal discretisation.

Here, we use a linear continuous Galerkin method for velocity and pressure, with a control volume formulation used for all tracer fields, including turbulence and biological tracers. Discontinuous linear Galerkin discretisations have also been tested and work successfully. For further details see Piggott et al. (2008b, 2009).

\subsection{Boundary conditions}

The domain used is quasi-1-D, $100 \mathrm{~m}$ square in the horizontal with depths of either $1000 \mathrm{~m}$ for Station Papa and Bermuda, or $2000 \mathrm{~m}$ for Station India. This ensures that the maximum MLD is well above the lower boundary at all stations. The lateral boundaries have a Dirichlet condition applied to the velocity such that the vertical component is zero. The top and bottom surfaces also have this condition applied. Boundary conditions for the turbulent quantities are as described in Hill et al. (2012) and are Neumann conditions for both turbulent equations. The top surface cell is subjected to heat, momentum and salinity fluxes. These are derived via the Large and Yeager (2004) bulk formulae, with atmospheric data supplied from ERA40 (Uppala et al., 2005). Both Station Papa and Station India use atmospheric forcing from 1970 onwards as this is when most observation data from those stations are available. Bermuda uses atmospheric forcing from 1980 onwards, again as most observational data was available during this period. Briefly, the three surface kinematic fluxes calculated - heat, $\langle w \theta\rangle$; salt, $\langle w s\rangle$; and momentum, $\langle w u\rangle$ and $\langle w v\rangle$ - can be related to the surface fluxes of heat $Q$, the freshwater $F$ and the momentum $\boldsymbol{\tau}=\left(\tau_{u}, \tau_{v}\right)$ via

$$
\begin{aligned}
& \langle w \theta\rangle=Q\left(\rho C_{p}\right)^{-1} \\
& \langle w s\rangle=F\left(\rho^{-1} S_{0}\right) \\
& (\langle w u\rangle,\langle w v\rangle)=\boldsymbol{\tau} \rho^{-1}=\left(\tau_{u}, \tau_{v}\right) \rho^{-1},
\end{aligned}
$$

where $\rho$ is the ocean density, $C_{p}$ is the heat capacity $\left(4000 \mathrm{~J} \mathrm{~kg}^{-1} \mathrm{~K}^{-1}\right.$ ) and $S_{0}$ is a reference ocean salinity, which is the current sea surface salinity. These fluxes are then 
applied as upper-surface Neumann boundary conditions on the appropriate fields.

\subsection{Biogeochemical model}

The model used here is a six-component model similar to the globally applicable model of Popova et al. (2006). Heuristically, the model consists of nutrients (ammonium and nitrate) which are fixed by phytoplankton in the presence of sunlight (photosynthetic active radiation - PAR). Phytoplankton shading is included, reducing the amount of PAR down the water column. Zooplankton grazes on phytoplankton and detritus partially recycling them back into inorganic nutrients and partially converting into detritus. Phytoplankton and zooplankton mortality also produce detritus which is gradually converted back to nutrients as it sinks through the water column.

Nutrients (ammonium and nitrate), detritus, phytoplankton and zooplankton are solved prognostically, whilst chlorophyll is a diagnostic variable, derived from phytoplankton. Initial conditions for phytoplankton, detritus and zooplankton concentrations were the same for all stations and are $0.1 \mathrm{mmol} \mathrm{m}^{-3}$ above $100 \mathrm{~m}$ and 0.005 below $100 \mathrm{~m}$. Ammonia was set to an initial value of $0.01 \mathrm{mmol} \mathrm{m}^{-3}$. Nitrate profiles were taken from Kleypas and Doney (2001) for Station Papa and Bermuda and Popova et al. (2006) for station India. For more details of this model see Appendix A1, which includes all parameters used. Note the parameters were tuned to give a good fit to all stations presented here, but were not altered between any model runs.

\subsection{Vertical turbulence model}

The generic length scale (GLS) turbulence parameterisation simulates vertical turbulence at a finer than that of the mesh. As the GLS model is a RANS parameterisation there is no dependency on the mesh resolution, provided the advective model simulates no turbulent processes, so is ideal for adaptive ocean-scale problems. GLS has the additional advantage that it can be set up to behave as a number of classical turbulence models: $k-\epsilon, k-k l, k-\omega$, and an additional model based on Umlauf and Burchard (2003), the gen model. The GLS model has been implemented within Fluidity and shown to work well with adaptive remeshing (Hill et al., 2012). Here, we use it in $k-\epsilon$ mode as detailed in Hill et al. (2012), which is a two-equation model. The first equation deals with the turbulent kinetic energy, $k$ :

$$
\frac{\partial k}{\partial t}+\boldsymbol{u}_{i} \frac{\partial k}{\partial x_{i}}=\frac{\partial}{\partial z}\left(\frac{v_{M}}{\sigma_{k}} \frac{\partial k}{\partial z}\right)+P+B-\epsilon
$$

where $\sigma_{k}$ is the turbulence Schmidt number for $k$, and $P$ and $B$ represent production by shear and buoyancy which are defined as

$$
\begin{aligned}
P & =-\overline{u^{\prime} w^{\prime}} \frac{\partial u}{\partial z}-\overline{v^{\prime} w^{\prime}} \frac{\partial v}{\partial z}=v_{M} M^{2} \\
M^{2} & =\left(\frac{\partial u}{\partial z}\right)^{2}+\left(\frac{\partial v}{\partial z}\right)^{2}, \\
B & =-\frac{g}{\rho_{0}} \overline{\rho^{\prime} w^{\prime}}=-v_{H} N^{2} \\
N^{2} & =-\frac{g}{\rho_{0}} \frac{\partial \rho}{\partial z} .
\end{aligned}
$$

Here, $N$ is the buoyancy frequency, $v_{M}$ is the kinematic eddy viscosity and $v_{H}$ is the kinematic eddy diffusivity, given by:

$v_{M}=\sqrt{k} l S_{M}+v_{M}^{0}, \quad v_{H}=\sqrt{k} l S_{H}+v_{H}^{0}$.

$\nu_{H}^{0}$ is the background diffusivity (set to $1 \times 10^{-6} \mathrm{~m}^{2} \mathrm{~s}^{-1}$ ), $v_{M}^{0}$ is the background viscosity $\left(1 \times 10^{-6} \mathrm{~m}^{2} \mathrm{~s}^{-1}\right), S_{M}$ and $S_{H}$ are often referred to as stability functions, $k$ is the turbulent kinetic energy, and $l$ is a length scale. When using GLS the values of $v_{M}$ and $v_{H}$ become the vertical components of the tensors $\boldsymbol{v}$ and $\boldsymbol{\kappa}_{T}$ in Eqs. (1) and (3) respectively. Other tracer fields, such as salinity use the same diffusivity as temperature, i.e. $\kappa_{T}=\kappa_{S}$.

There is also the option to add an extra term to account for additional oceanic physics, such an internal waves breaking. This is based on the NEMO ocean model (Madec, 2008) and takes a user-defined percentage of the surface $k$ and distributes it down-depth using an exponential profile:

$k(z)=k_{0}(z)+\alpha k_{\text {sur }} \exp \left(-z / l_{k}\right)$,

where $k$ is the new turbulent kinetic energy value at depth $z$, $k_{0}$ is the original turbulent kinetic energy, $k_{\text {sur }}$ is the surface turbulent kinetic energy, $\alpha$ is a constant for the amount (percentage) of surface turbulent kinetic energy to transfer down the column, and $l_{k}$ is a length scale (m) over which this decay occurs. In this work, $\alpha=0.05$ and $l_{k}=30$.

The second equation gives the dissipation $\epsilon$, which is described by

$$
\frac{\partial \epsilon}{\partial t}+\boldsymbol{u}_{i} \frac{\partial \epsilon}{\partial x_{i}}=\frac{\partial}{\partial z}\left(\frac{v_{M}}{\sigma_{\epsilon}} \frac{\partial \epsilon}{\partial z}\right)+\frac{\epsilon}{k}\left(c_{1} P+c_{3} B-c_{2} \epsilon\right),
$$

where $c_{1}, c_{2}, c_{3}$ and $\sigma_{\epsilon}$ are constants with values given by Hill et al. (2012).

The MLD can be defined in a number of ways. Here, we use two definitions: (1) where $k<1 \times 10^{-5} \mathrm{~m}^{2} \mathrm{~s}^{-2}$ and (2) where density is $0.125 \mathrm{~kg} \mathrm{~m}^{-3}$ less than surface density (i.e. at $z=0$ ). We use the second when comparing to observational data and the first for determining weighting of the $k$ field when using adaptivity (see next section).

\subsection{Dynamic adaptive mesh optimisation}

The mesh adaptivity algorithm used in this work attempts to optimise the size as well as the shape of individual elements 
of the mesh in order to minimise an optimisation functional (Pain et al., 2001; Piggott et al., 2005, 2008b). We use the interpolation error which is often a reasonable indication of the error due to spatial discretisation in finite-element problems (Pichelina et al., 2000). In Fluidity, mesh adaptivity aims to increase resolution in regions of the domain with large curvatures of given fields and decrease resolution elsewhere. This approach allows good representation of the small-scale dynamics and sharp gradients without the need for high spatial resolution throughout the entire domain (Piggott et al., 2005). The mesh is adapted through a series of local topological and geometrical operations as described by Pain et al. (2001). In this work we adapt in the vertical direction only. A single column of mesh vertices is first adapted. This column is then replicated to the other three columns, which are then joined to form a quasi-1-D column of tetrahedra. The location of the vertices is constructed such that all elements in that first 1-D column have unit edge length when measured with respect to a given adaptivity metric, $M$.

In Fluidity a relatively simple adaptivity metric $M$ is employed. For chosen fields $f_{i}$, adaptivity metrics $M_{i}$ are defined by

$M_{i}=\operatorname{det}\left|H_{i}\right|^{-\frac{1}{2 p+n}} \frac{\left|H_{i}\right|}{\varepsilon_{i}}$,

where $\varepsilon_{i}$ is a user-defined weight corresponding to the field under consideration and $\left|H_{i}\right|$ is the Hessian matrix (secondorder derivatives) for that field where the absolute values of its eigenvectors have been taken, $p \in \mathbb{Z}$ and $n$ is the dimension of the space (Loseille and Alauzet, 2011). The Hessian matrix contains information about both the magnitude and direction of the curvature of a field and hence can be used to guide generation of anisotropic elements. The final adaptivity metric used, $M$, is formed from a superposition of the adaptivity metrics for individual fields: $M=\bigcup_{i} M_{i}$ (Pain et al., 2001). In the work presented here we tested values of $p$ of 2 and $\infty$ as both have been used in previous work, but $p=2$ has shown superior results in resolving both weak and strong curvatures simultaneously within the same simulation (Loseille and Alauzet, 2011; Hiester et al., 2011) and is used in all simulations presented. $\varepsilon$ may be varied spatially and temporally, but neither is utilised here. In general, for a given solution field, decreasing $\varepsilon$ will lead to greater refinement of the mesh and increasing $\varepsilon$ will lead to more coarsening. At this point the adaptivity metric is also modified to take into account bounds upon the maximum and minimum element size, maximum allowable aspect ratio, edge length gradation, and number of elements. For more details see Pain et al. (2001); Piggott et al. (2005, 2008b); Hiester et al. (2011) and references therein.

The mesh is adapted at run time and the frequency with which it adapts can also be specified. Here, we specify an adapt frequency of $5 \mathrm{~h}$. After an adapt the solution fields must be interpolated from the pre- to post-adapt meshes. Two methods are available: "linear-interpolation" and "bounded
Galerkin-projection" (Farrell et al., 2009). All prognostic fields are interpolated, along with any diagnostic fields as required. Three different interpolation methods were tested in this work: linear interpolation, which is bounded but nonconservative; Galerkin projection, which is conservative and can be made bounded at the expense of a minimal amount of diffusion (Farrell et al., 2009); and a mixture of the two, in which Galerkin projection was used for biological tracers, and linear interpolation was used for physical quantities. It is anticipated that conservation of the integral of biological quantities is crucial to obtaining a satisfactory solution, but that the physical quantities - velocity, temperature and salinity - only require linear interpolation (Hill et al., 2012). As linear interpolation is less computationally demanding than Galerkin projection, further savings in computational cost over and above those obtained through those obtained through the use of adaptivity can be gained using linear interpolation where it is adequate.

The adaptivity metric used to alter the mesh is crucial to obtaining an optimal simulation (Hiester et al., 2011). Here, we test four different adaptivity metric formulations which govern element sizes: PAR, Bio, Bio and Phys, and Phys. These use the photosynthetic active radiation only (PAR), biological fields and photosynthetic active radiation (Bio), physical fields only (Phys) or a combination (Bio and Phys). The same adaptivity metrics are used for all three test stations as we are attempting to provide an adaptivity metric formulation that works well in a variety of ocean settings and to avoid "tweaking" of the adaptivity metric for a particular location. The physical fields used are the density and velocity, and the biological fields used are the nutrients and PAR. Details of the fields used and the weighting of each field are given in Table 1. It is important to note that Fluidity allows a great deal of flexibility in choosing the adaptivity metric, unlike in the previous studies described above. Here, we investigate how the choice of which fields (physical and biological) are included in the adaptivity metric affects a simulation. We do not investigate the effects of changing the user-defined weights $\varepsilon$; they are chosen to give a reasonable result and may not be optimal. For the purposes of this paper $\varepsilon$ being sub-optimal is not critical. The weights were chosen based on a preliminary parameter study and give a good compromise between good results and minimal element numbers.

The aim of choosing these fields is to enable tracking of the MLD variation on both a daily and seasonal level. This will concentrate resolution only where changes of the above quantities are large, minimising the number of elements elsewhere. The physical fields chosen are density and velocity. Density will show a steep vertical change at the base of the mixed layer, whilst velocity varies strongly in the top few metres of the water column. However, if winds strengthen, more resolution will be added as the mixing deepens. The biological tracers chosen are the nutrients and PAR. Fluxes of nutrients from depth are the main cause of plankton blooms at both India and Bermuda. However, the nutrient high, and 
Table 1. Weighting of fields used for each metric used in this study. A “-” indicates that this field was not used in the metric construction. See Eq. (15).

\begin{tabular}{lcccc}
\hline Metric & Nutrient $(\varepsilon)\left(\mathrm{mmol} \mathrm{m}^{-3}\right)$ & PAR $(\varepsilon)(\mathrm{W} \mathrm{m}-2)$ & Velocity $(\varepsilon)\left(\mathrm{m} \mathrm{s}^{-1}\right)$ & Density $(\varepsilon)\left(\mathrm{kg} \mathrm{m}^{-3}\right)$ \\
\hline Bio & 10.0 & 0.1 & - & - \\
Phys & - & - & $(0.1,0.1,10.0)$ & 0.01 \\
Bio and Phys & 10.0 & - & $(0.1,0.1,10.0)$ & 0.01 \\
PAR & - & 0.1 & - & - \\
\hline
\end{tabular}

hence phytoplankton high, may not be at the surface; this is the case in Bermuda where there is a significant subsurface chlorophyll maximum. By resolving the nutrient fluxes closely we aim to also then resolve the other biological tracers as a consequence. In contrast, Station Papa shows only weakly varying surface nutrient changes. However, the upward flux of nutrients can lead to erroneous timings of the spring bloom. Therefore, the base of the MLD shows a substantial vertical nutrient change and hence adding resolution here should minimise spurious vertical numerical diffusivity. PAR is important only in the top $100 \mathrm{~m}$ of the domain and varies daily and hence adding this field to the adaptivity metric will add extra resolution during daylight hours down to the bottom of the photic zone.

To examine the effect of adaptive remeshing on biogeochemical models we perform a number of experiments. For each station we run fixed mesh simulations at different resolutions to examine the effects of vertical mesh resolution on the biogeochemistry. We qualitatively compare the results from the highest fixed-mesh resolution to available observations at each station. We then perform experiments varying the adaptivity metric at each station and evaluate the results compared to the highest resolution fixed-mesh simulation using the root mean square error of a number of fields to quantitatively evaluate the performance. Finally, we perform two specific examples tracking other biological tracers to demonstrate possible uses for the adaptive remeshing technology.

\section{Model evaluation}

We first examine a single fixed-mesh case for each station, comparing them to available observational data from Kleypas and Doney (2001) and Popova et al. (2006), before showing that the simulated response depends on the model's vertical resolution.

Station Papa in the Northwest Pacific is an ideal testing station for a 1-D simulation. There is little horizontal advection, and as such, Papa has been used to assess numerical models (Denman and Miyake, 1973; Burchard and Bolding, 2001; Hill et al., 2012). Fluidity has also been previously shown to work well at replicating the expected physics with adaptive meshes here (Hill et al., 2012). Papa's distinguishing feature is that nutrients are not limited and hence surface nutrients exhibit only a small seasonal variation. Surface
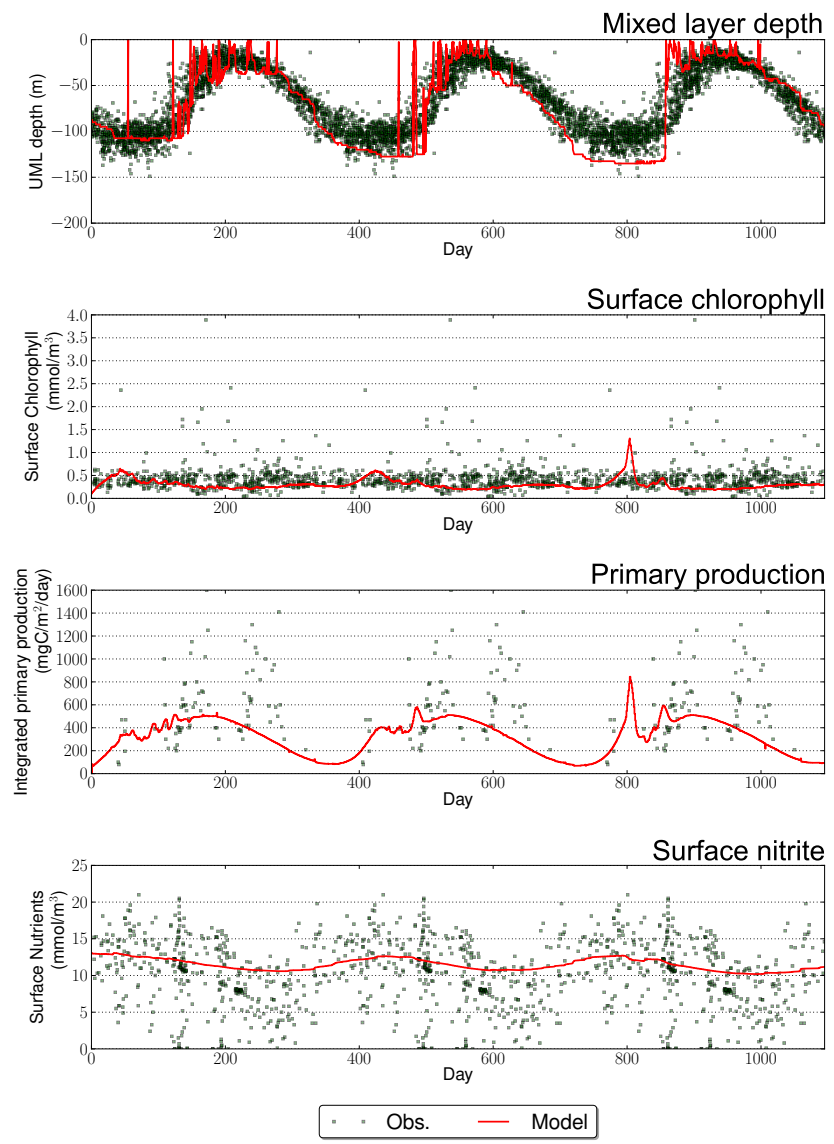

Fig. 2. Summary of simulated physical and biological behaviour at Station Papa for a uniform (2.5 m), non-adaptive simulation. From top to bottom, panels show MLD, surface chlorophyll, integrated primary productivity and surface nutrients. Where available, measured data are shown as green squares. Measured data are plotted against day of year due to lack of data for some quantities.

values are those occurring on the uppermost element, which should be the same throughout the MLD. The results of the biogeochemical model show reasonable agreement with measured data (Fig. 2), replicating the major features of this station. The surface nutrients show the desired dip over the summer months, though this is not quite as pronounced as the observed data. The surface chlorophyll shows good 

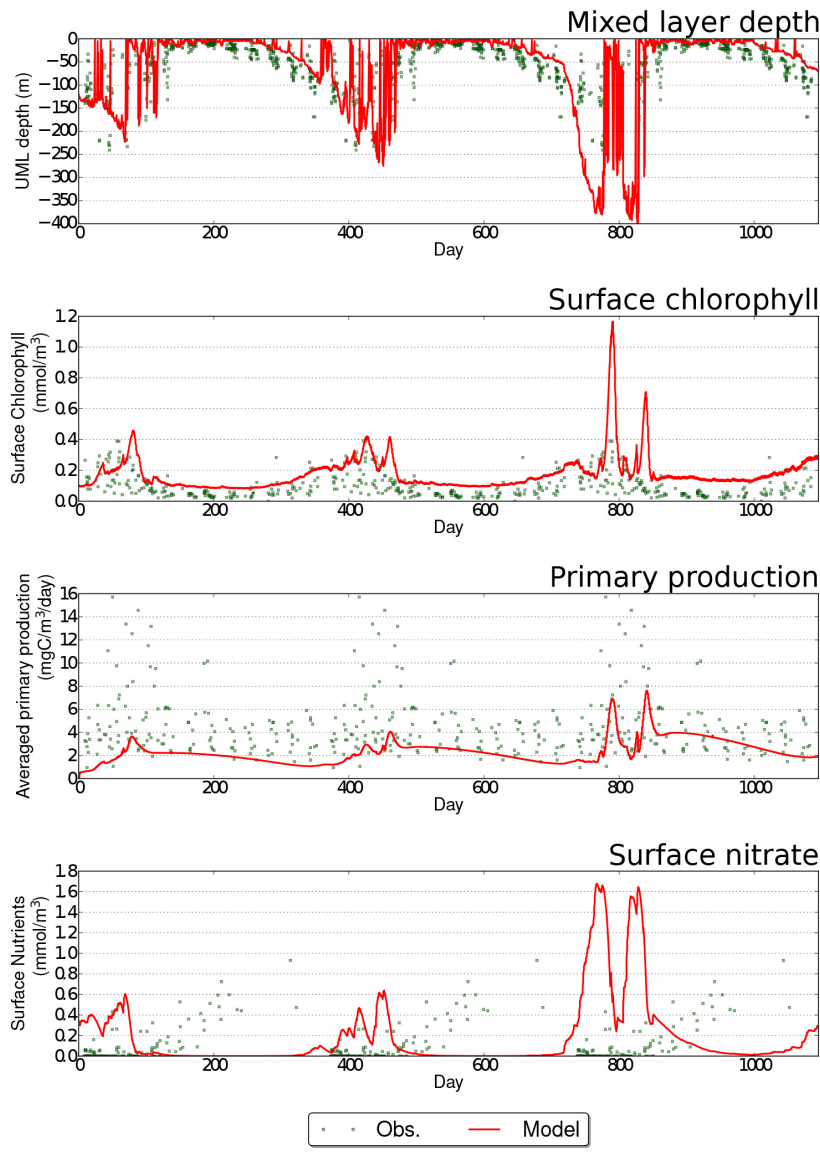

Fig. 3. Summary of simulated physical and biological behaviour at Bermuda for a uniform $(2.5 \mathrm{~m})$, non-adaptive simulation. From top to bottom, panels show MLD, surface chlorophyll, averaged primary productivity and surface nutrients. Where available, measured data are shown as green squares. Measured data are plotted against day of year due to lack of data for some quantities.

agreement to observed data, as does the integrated primary production (note: this is integrated over the mixed layer).

The model result at Bermuda, unlike Papa, shows some differences to the measured data (Fig. 3). The surface nutrient shows the observed nutrient-limited behaviour, but the limiting of nutrients occurs too early in the year. The third winter (days 700-900) shows a marked deepening of the mixed layer. This is due to surface forcing particular to that year, and longer simulations (not shown) show a return to the more normal behaviour seen in years one and two. Surface chlorophyll values lie on the upper limit of observed data, with a small peak in the spring. The primary production (note: averaged over MLD) is around a factor of two too low. However, given that we are simulating an isolated 1-D column, without any horizontal transport of quantities in or out of the domain, we believe this is a reasonable result. There is a substantial subsurface chlorophyll maximum (Fig. 4) as has been shown in measured observation and it is of similar magnitude to that

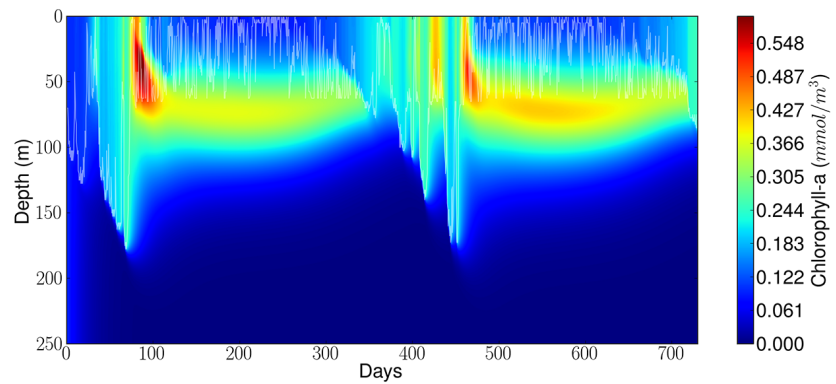

Fig. 4. Time-depth plot of chlorophyll at Bermuda, showing the clear subsurface chlorophyll maxima.

obtained in previous modelling studies (e.g. Anderson and Pondaven, 2003).

Unlike for the previous stations there is a lack of MLD data for Station India. However, the model again gives a sufficiently good match to available data, although with larger discrepancies compared to other stations (Fig. 5). The spring bloom (as shown by the surface chlorophyll) happens around 30 days early, with a peak that is perhaps a factor of four too high. Similarly, the integrated primary production (note: integrated over the mixed layer) shows a peak of around 2-3 times the observed value at the same time. However, the values during the rest of the year lie around the lower limit of observed data. Surface nutrients show reasonable agreement with the timing of the spring decrease, but the level is perhaps a factor of two too high during the summer months.

\subsection{Resolution dependence}

For all stations we have run the simulations on a number of fixed meshes, varying the vertical resolution between $20 \mathrm{~m}$ and $2.5 \mathrm{~m}$. We use the highest resolution $(2.5 \mathrm{~m})$ fixed-mesh simulation as "truth" when assessing the performance of the adaptive mesh simulations. In addition, we use qualitative comparisons to observational data at each station to ensure that the model performs as expected, given the lack of horizontal dynamics.

A standard root mean square (rms) error was used to assess model performance. The rms error is calculated as

$\epsilon_{\mathrm{rms}}=\sqrt{\frac{\sum_{i=1}^{n}\left(x_{i}-y_{i}\right)^{2}}{n}}$,

where $x_{i}$ is the quantity being assessed in the high-resolution simulation and $y_{i}$ is the value of the quantity produced by the simulation being considered. All simulations were run for three years. Output for all runs was produced every $12 \mathrm{~h}$, giving $n=2190$. For each field of interest a separate $\epsilon_{\mathrm{rms}}$ is calculated, giving $\epsilon_{\mathrm{MLD}}$ for the MLD. For biological quantities the $\mathrm{L} 2_{\text {norm }}$ of the water column was used, giving $\epsilon_{\mathrm{N}}$ for 

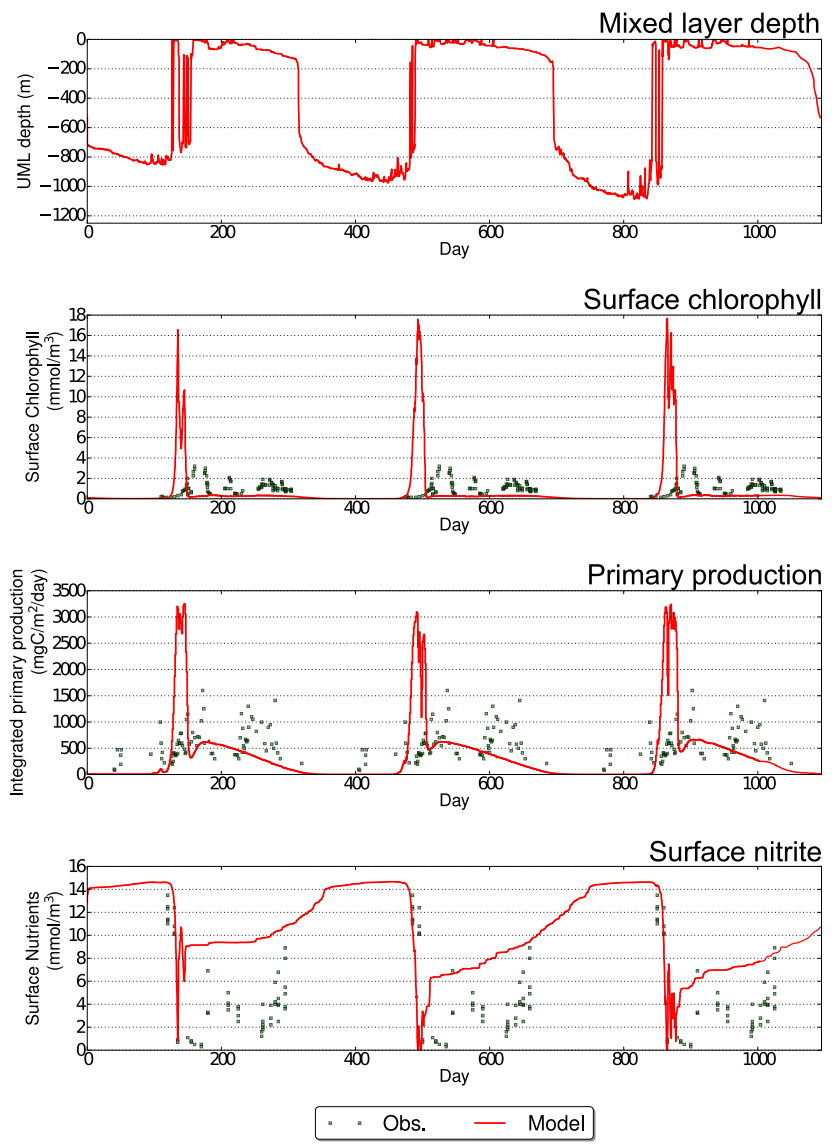

Fig. 5. Summary of simulated physical and biological behaviour at Station India for a uniform $(2.5 \mathrm{~m})$, non-adaptive simulation. From top to bottom, panels show MLD, surface chlorophyll, integrated primary productivity and surface nutrients. Where available, measured data are shown as green squares. Measured data are plotted against day of year due to lack of data for some quantities.

nutrients, $\epsilon_{\mathrm{P}}$ for primary productivity, $\epsilon_{\mathrm{C}}$ for chlorophyll and $\epsilon_{\mathrm{Z}}$ for zooplankton. Here, the $\mathrm{L} 22_{\text {norm }}$ is defined as

$\mathrm{L} 2_{\text {norm }}=\sqrt{\int_{0}^{n}|S|^{2} \mathrm{~d} V,}$

where $V$ is the volume of the domain and $S$ is the scalar field in question.

Figures $6-8$ show a single year (year 2 of the 3 -year simulation to allow for model spin-up) for each station. For Station Papa (Fig. 6) there is a noticeable difference in MLD behaviour with higher resolutions showing deeper winter mixing. This in turn affects the upward mixing of nutrients, which show a marked jump when resolution is refined from 10 to $5 \mathrm{~m}$. Both 5 and $2.5 \mathrm{~m}$ resolutions show broadly similar patterns. The difference in upward mixing of nutrients then affects the primary productivity, and surface chlorophyll shows a difference in peak surface chlorophyll of around
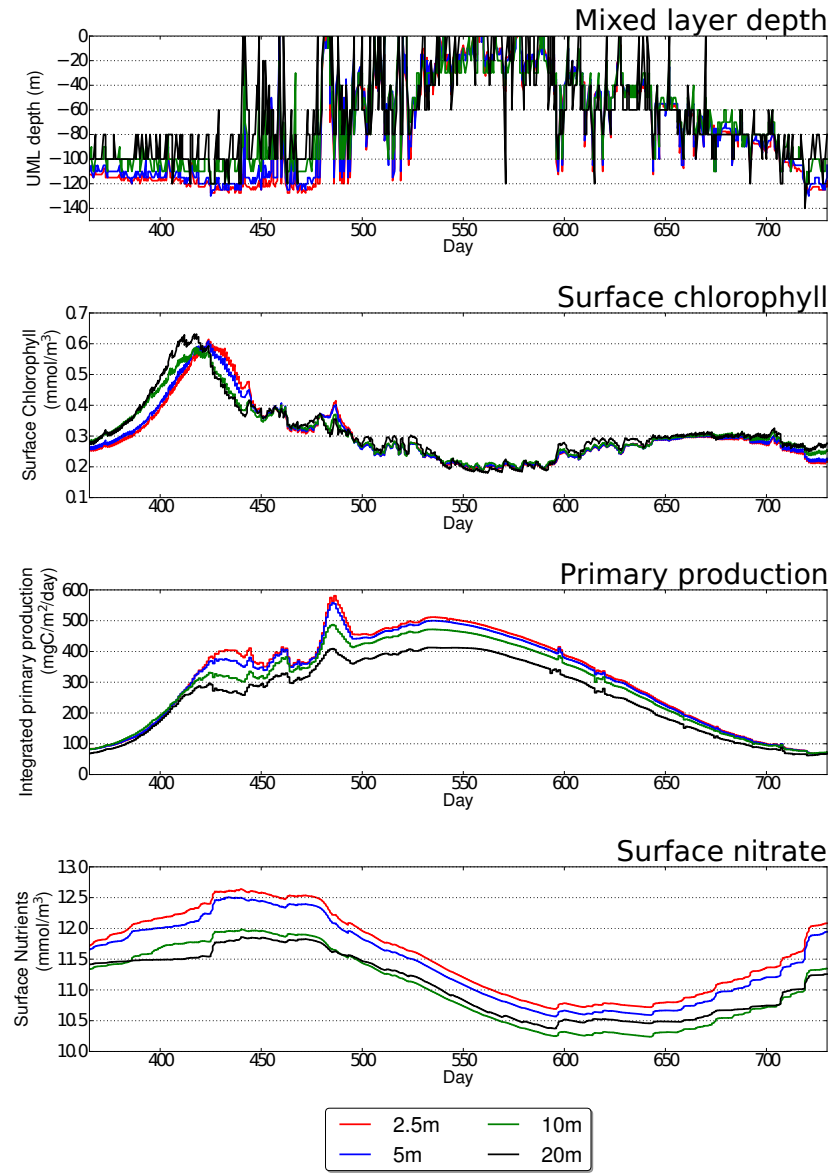

Fig. 6. Summary of simulated physical and biological behaviour at Station Papa using uniform meshes at a number of resolutions. From top to bottom, panels show MLD, surface chlorophyll, integrated primary productivity and surface nutrients.

20 days. Bermuda shows a similar pattern (Fig. 7) with increased resolution producing higher peak nutrients due to increased upmixing, which in turn leads to increased surface chlorophyll. Finally, Station India (Fig. 8) shows a marked increase in primary productivity within the MLD, which is doubled when resolution is refined from 20 to $2.5 \mathrm{~m}$. The surface nutrient data show little difference with resolution, so a sensible interpretation is that this is due to the slight increase in summer MLD depths with increased resolution. It is therefore clear that all stations show a response to vertical resolution which is the result of a complex interaction between the mixing caused by the vertical turbulence model and the biological sources and sinks.

Resolution dependence can also be seen in vertical profiles. Chlorophyll vertical profiles were taken at day 547 (mid-summer of the second year) and day 730 (mid-winter at the end of the second year) (Fig. 9). Each station shows a change in the simulated value with increasing resolution. This is perhaps most pronounced at Station Papa during the winter, where the MLD increases from around 100 
Table 2. The rms error, $\epsilon$, of fixed-mesh simulations compared to the simulation with $2.5 \mathrm{~m}$ vertical resolution at Station Papa. $\epsilon$ is shown for MLD, and the L2 2 orm of nutrient, primary productivity, chlorophyll and zooplankton. See Fig. 6 also.

\begin{tabular}{lrrrrrr}
\hline Res $(\mathrm{m})$ & $\epsilon_{\mathrm{MLD}}(\mathrm{m})$ & $\epsilon_{\mathrm{N}}\left(\mathrm{mmol} \mathrm{m}^{-3}\right)$ & $\epsilon_{\mathrm{P}}\left(\mathrm{mmol} \mathrm{m}^{-3}\right)$ & $\epsilon_{\mathrm{C}}\left(\mathrm{mmol} \mathrm{m}^{-3}\right)$ & $\epsilon_{\mathrm{Z}}\left(\mathrm{mmol} \mathrm{m}^{-3}\right)$ & No. elements \\
\hline $5 \mathrm{~m}$ & 5.244 & 0.119 & 0.098 & 0.0095 & 0.0045 & 1200 \\
$10 \mathrm{~m}$ & 10.640 & 0.506 & 0.342 & 0.0445 & 0.0169 & 600 \\
$20 \mathrm{~m}$ & 16.521 & 0.421 & 0.628 & 0.0486 & 0.0283 & 300 \\
\hline
\end{tabular}

Table 3. The rms error, $\epsilon$, of fixed-mesh simulations compared to the simulation with $2.5 \mathrm{~m}$ vertical resolution at Bermuda. $\epsilon$ is shown for MLD, and the L2norm of nutrient, primary productivity, chlorophyll and zooplankton. See Fig. 7 also.

\begin{tabular}{lrrrrrr}
\hline Res $(\mathrm{m})$ & $\epsilon_{\mathrm{MLD}}(\mathrm{m})$ & $\epsilon_{\mathrm{N}}\left(\mathrm{mmol} \mathrm{m}^{-3}\right)$ & $\epsilon_{\mathrm{P}}\left(\mathrm{mmol} \mathrm{m}^{-3}\right)$ & $\epsilon_{\mathrm{C}}\left(\mathrm{mmol} \mathrm{m}^{-3}\right)$ & $\epsilon_{\mathrm{Z}}\left(\mathrm{mmol} \mathrm{m}^{-3}\right)$ & No. elements \\
\hline $5 \mathrm{~m}$ & 7.079 & 0.408 & 0.140 & 0.0129 & 0.0113 & 1200 \\
$10 \mathrm{~m}$ & 12.630 & 0.402 & 0.149 & 0.0160 & 0.0236 & 600 \\
$20 \mathrm{~m}$ & 18.710 & 0.397 & 0.226 & 0.0285 & 0.0410 & 300 \\
\hline
\end{tabular}
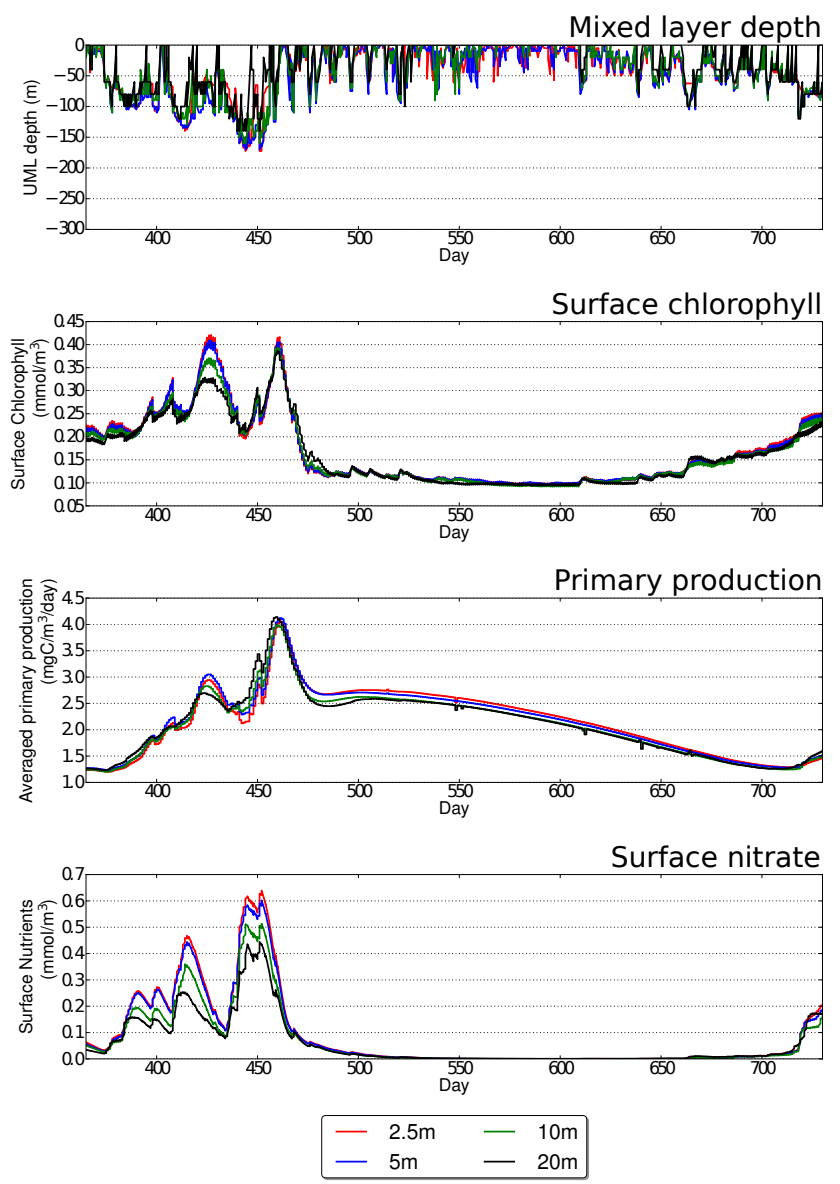

Fig. 7. Summary of simulated physical and biological behaviour at Bermuda using uniform meshes at a number of resolutions. From top to bottom, panels show MLD, surface chlorophyll, MLD averaged primary productivity and surface nutrients.
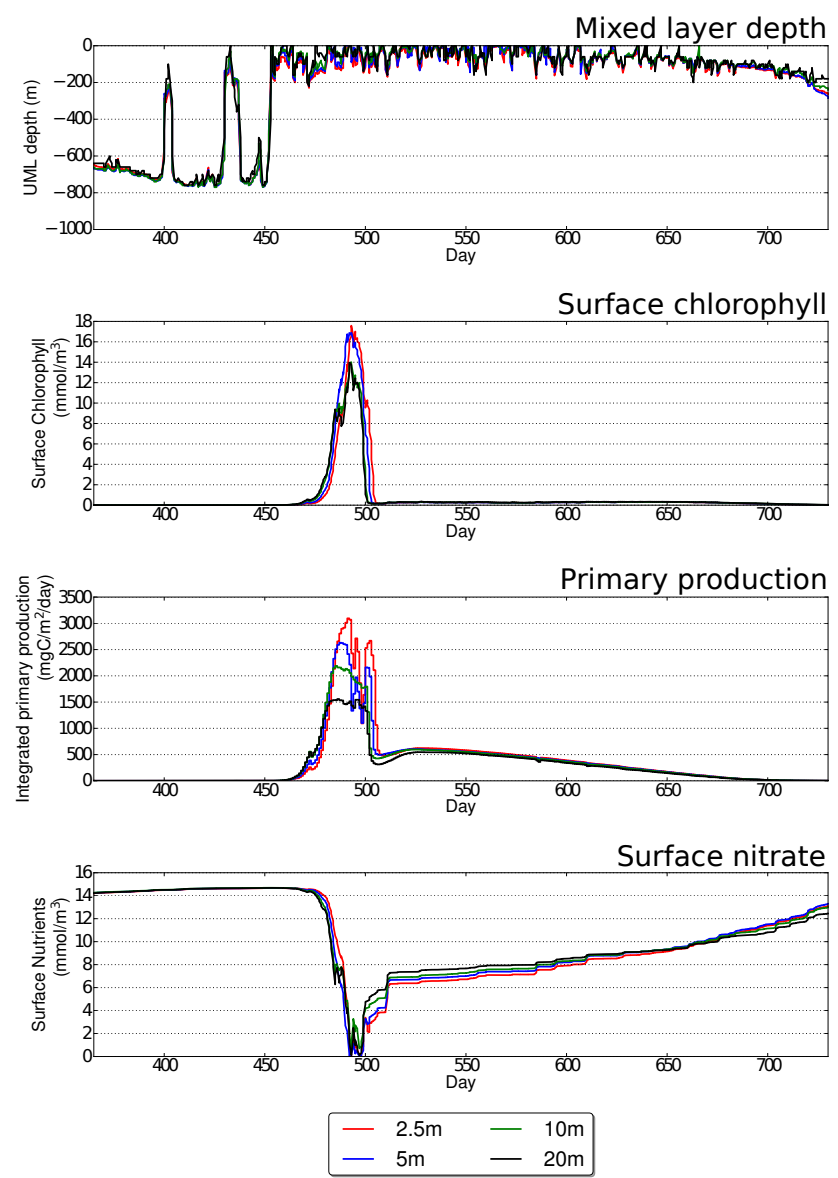

Fig. 8. Summary of simulated physical and biological behaviour at Station India using uniform meshes at a number of resolutions. From top to bottom, panels show MLD, surface chlorophyll, integrated primary productivity and surface nutrients. 
Table 4. The rms error, $\epsilon$, of fixed-mesh simulations compared to the simulation with $2.5 \mathrm{~m}$ vertical resolution at Station India. $\epsilon$ is shown for MLD, and the L2 2 norm of nutrient, primary productivity, chlorophyll and zooplankton. See Fig. 8 also.

\begin{tabular}{lrrrrrr}
\hline Res $(\mathrm{m})$ & $\epsilon_{\mathrm{MLD}}(\mathrm{m})$ & $\epsilon_{\mathrm{N}}\left(\mathrm{mmol} \mathrm{m}^{-3}\right)$ & $\epsilon_{\mathrm{P}}\left(\mathrm{mmol} \mathrm{m}^{-3}\right)$ & $\epsilon_{\mathrm{C}}\left(\mathrm{mmol} \mathrm{m}^{-3}\right)$ & $\epsilon_{\mathrm{Z}}\left(\mathrm{mmol} \mathrm{m}^{-3}\right)$ & No. elements \\
\hline $5 \mathrm{~m}$ & 137.03 & 1.04 & 303.258 & 1.538 & 0.222 & 2400 \\
$10 \mathrm{~m}$ & 138.18 & 1.21 & 357.834 & 1.770 & 0.247 & 1200 \\
$20 \mathrm{~m}$ & 142.71 & 1.50 & 455.565 & 1.992 & 0.264 & 600 \\
\hline
\end{tabular}

to nearly $120 \mathrm{~m}$ when resolution is increased from 20 to $2.5 \mathrm{~m}$. Bermuda shows a decrease of both winter and summer subsurface chlorophyll maxima with increasing resolution. These vertical profiles show that the model is numerically stable, producing adequate results at even low resolution, but that vertical resolution does affect the profile simulated.

The response to resolution can be examined more quantitatively using a simple convergence test. Although convergence is non-trivial for nonlinear dynamics (Hill et al., 2012), a decrease in error should be seen with increasing vertical resolution. For all stations there is clear convergence (a decrease in error) for the MLD (Tables 2-4). Ideally, for the set-up described previously, this should be at least first-order convergence. Both Bermuda and Station Papa show this behaviour but Station India does not (though there is still a decrease in error with increasing resolution). However, for most variables there is a decrease in the error measure compared to the highest resolution simulations at each station.

The surface nutrients error stays approximately constant at both Bermuda and Station India (Tables 3 and 4). Despite these exceptions there is a clear dependence on resolution, with higher resolutions generally matching the highest resolution simulation with higher accuracy. At Papa, all biological quantities bar nutrients show a general convergence in error as resolution is increased (Table 2). The error at $10 \mathrm{~m}$ vertical resolution appears to be double that expected, but there is a convergence in error from 10 to $5 \mathrm{~m}$. Bermuda shows clear first-order convergence of MLDs and zooplankton; and less certain convergence of chlorophyll (Table 3). Surface nutrient error appears to be constant, as does primary productivity (average over the MLD). Finally, Station India shows a general convergence with increasing resolution for all biological quantities, though not at first order (Table 4).

From these results we can see that there is a general decrease in error to the highest resolution run with increasing resolution. Therefore, using vertical adaptivity should allow a minimisation in the number of elements within the computational mesh whilst ensuring that error does not increase to an unreasonable level.

\section{Adaptivity}

We have carried out the same simulations as above using an adaptive mesh guided by a variety of different adaptivity metrics and, in addition, we have tested different interpolation

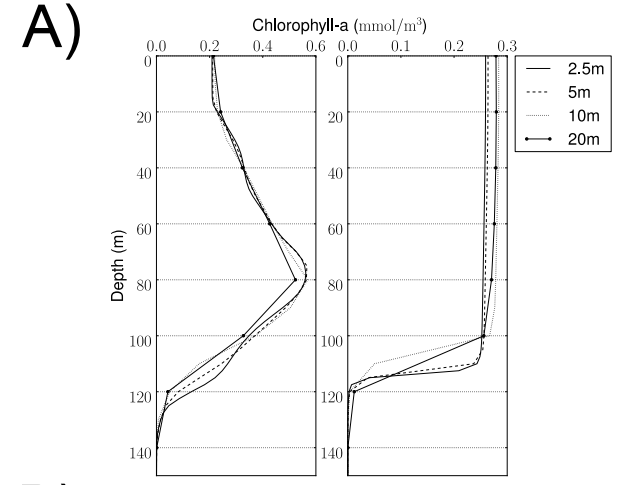

B)

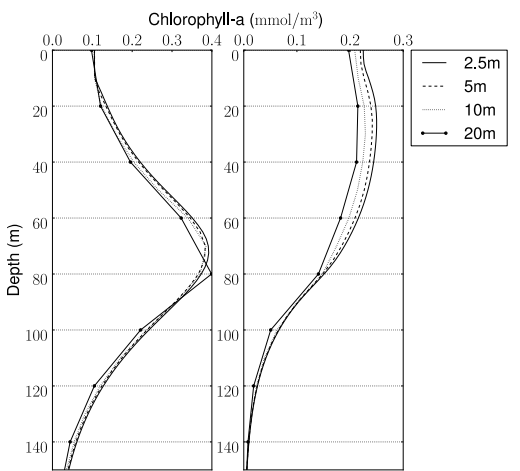

C)

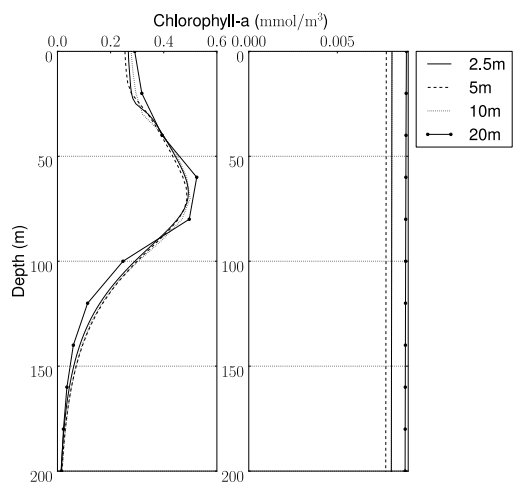

Fig. 9. Vertical profiles of chlorophyll at (A) Station Papa, (B) Bermuda and (C) Station India over the top 150, 150 and $200 \mathrm{~m}$ respectively using uniform meshes at a number of resolutions. The left-hand column shows the profile in summer (day 182) and the right-hand column shows the profile in winter (day 365). There are changes in values at key depths of up to $15 \%$ depending on the vertical resolution used. 
A)

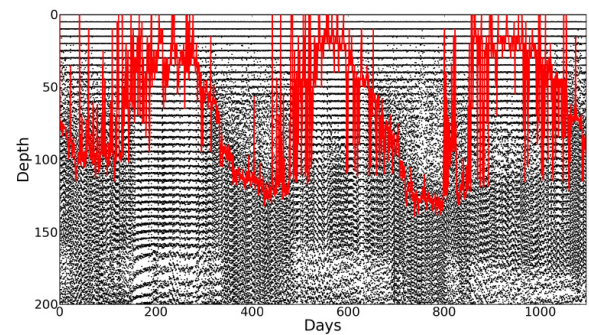

C)

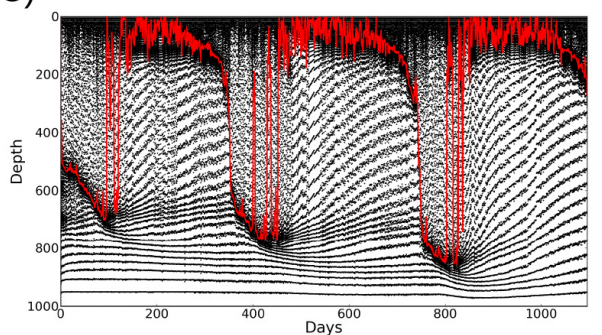

B)

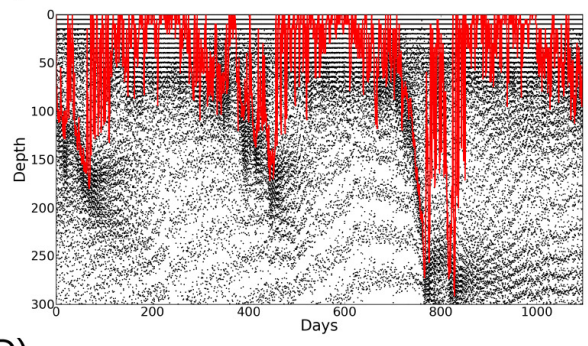

D)

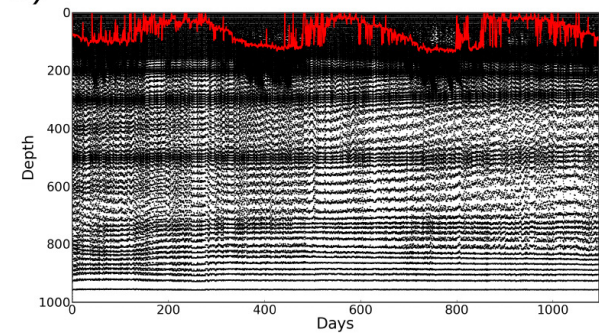

Fig. 10. Representation of the meshes obtained via adaptivity for all stations using the bio and phys metric. A dot is placed on each vertex in the mesh and this is repeated for each output time. Clustering of vertices therefore indicate higher resolution. Station Papa (A) shows reduced resolution under the mixed layer during summer, but high resolution persists for some distance below the mixed layer. Bermuda (B) shows substantial reduction of resolution below the mixed layer, but with the minimum resolution being maintained during the summer in the upper layers. Similarly, India (C) tracks the MLD, with decreased resolution within the mixed layer, whilst maintaining good resolution in the upper portion of the water column. Gradients in density mean that high-resolution zones are maintained at Station Papa (D) at up to $800 \mathrm{~m}$ depth.

methods at Station Papa. For simplicity, simulations at the three test station used the same adaptivity settings. Adaptivity was performed every $5 \mathrm{~h}$. This allows changes in ocean surface forcing (which has a temporal frequency of $6 \mathrm{~h}$ ) to be captured, along with diurnal fluctuations. Over a 3-year simulation a total of 5256 adapts are thus performed. This is a large number and therefore any additional numerical diffusivity or noise derived from adapting the mesh will be evident in the final simulation results when compared to the fixedmesh simulations. The minimum and maximum edge lengths permitted are set to 5 and $50 \mathrm{~m}$ respectively. We therefore hope to find that the adaptive simulations are equivalent to the $5 \mathrm{~m}$ fixed resolution simulations, but use substantially fewer elements.

The adaptive algorithm was performed on a single vertical column of mesh vertices and the position of these were replicated to the other three columns. In this way we obtained a layered mesh, with vertical resolution of the layers varying according to the chosen adaptivity metric and the simulated state at the time of the adapt. Apart from the adaptive mesh, the simulations were completely identical to the fixed-mesh simulations.

The meshes produced by the adaptive algorithm showed broadly similar features between different adaptivity metrics for each particular site (Fig. 10). Comparing those produced by the adaptivity metric using both the biological and physical fields shows the mesh tracking the behaviour of the MLD.
In addition, high resolution is maintained in the photic layer, but reduces with the mixed layer when the MLD increases substantially.

\subsection{Effect of interpolation method}

The tests at Station Papa (Fig. 11) show that Galerkin projection for all fields gives the best result for both physical behaviour (MLD) and biological quantities. In particular, there is a significant surface nutrient drift when using linear interpolation, and less so when using a mixed formulation. The effect is clear by day 1000 , where surface nutrients are $25 \%$ higher when using a mixed formulation and over $50 \%$ higher than the fixed-mesh simulation when using linear only. The cause of this drift is additional numerical diffusion due to the linear interpolation in the physical fields (mixed and linear only) and lack of conservation (linear only). Using Galerkin projection on both physical and biological fields gives a result that is almost identical to the fixed-mesh simulation. The MLD behaviour changes in response to the interpolation method used, with a marked shoaling of the MLD around day 800 when using linear or mixed interpolation. The mixed interpolation formulation performed only slightly better in this regard and using Galerkin projection on the turbulent parameterisation quantities only improved the solution slightly (results not shown). These results contrast to those presented by Hill et al. (2012), where linear interpolation was used and adaptivity performed well at Station Papa. 
Table 5. The rms error, $\epsilon$, of adaptive mesh simulations compared to the simulation with $2.5 \mathrm{~m}$ vertical resolution at Station Papa. $\epsilon$ is shown for MLD, and the L2 2 orm of nutrient, primary productivity, chlorophyll and zooplankton. See Fig. 6 also.

\begin{tabular}{lrrrrrr}
\hline Res $(\mathrm{m})$ & $\epsilon_{\mathrm{MLD}}(\mathrm{m})$ & $\epsilon_{\mathrm{N}}\left(\mathrm{mmol} \mathrm{m}^{-3}\right)$ & $\epsilon_{\mathrm{P}}\left(\mathrm{mmol} \mathrm{m}^{-3}\right)$ & $\epsilon_{\mathrm{C}}\left(\mathrm{mmol} \mathrm{m}^{-3}\right)$ & $\epsilon_{\mathrm{Z}}\left(\mathrm{mmol} \mathrm{m}^{-3}\right)$ & No. elements $($ Mean, min, max $)$ \\
\hline $5 \mathrm{~m}$ & 5.244 & 0.119 & 0.0986 & 0.0095 & 0.0045 & 1200 \\
\hline Bio and Phys & 4.769 & 0.053 & 0.385 & 0.0038 & 0.0020 & $458.5,372,558$ \\
Bio Only & 14.211 & 0.589 & 0.529 & 0.0426 & 0.0300 & $386.1,336,450$ \\
Phys Only & 4.837 & 0.070 & 0.398 & 0.0034 & 0.0021 & $310.1,240,408$ \\
PAR & 23.324 & 2.664 & 0.592 & 0.0790 & 0.0330 & $612.2,532,660$ \\
\hline
\end{tabular}
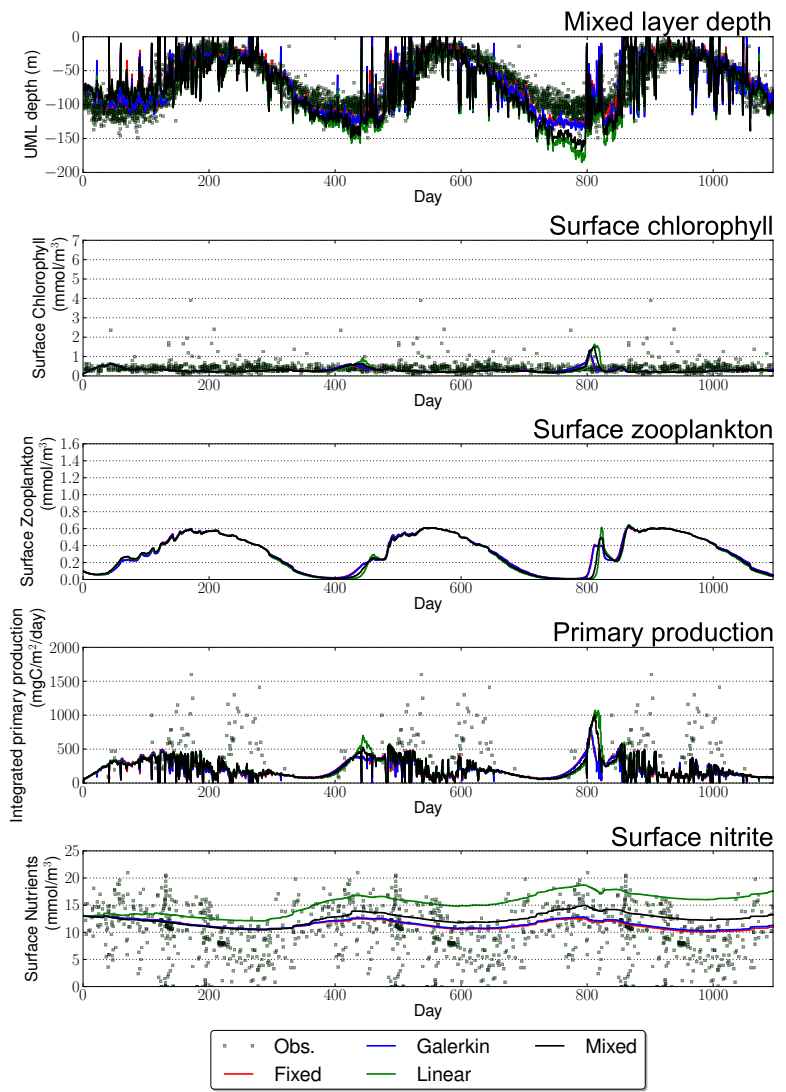

Fig. 11. Summary of simulation results from Station Papa comparing the fixed high-resolution $(2.5 \mathrm{~m})$ simulation with adaptive simulation using different interpolation methods between meshes. Linear and mixed perform poorly, inducing extra vertical diffusivity, compared to Galerkin projection. Panels and data are the same as in Fig. 6.

However, here we have added the additional term to simulate internal wave breaking (Eq. 13) and the adaptivity metric in Hill et al. (2012) was tuned to Station Papa only, with a lower minimum edge length. It is also worth noting that the temperature and salinity fields showed little or no difference between the fixed and adaptive simulations as was shown in Hill et al. (2012); it is the biological tracers that highlight undesired behaviour, i.e. upward fluxing of tracers, of the adaptive runs. Galerkin projection is therefore used for all subsequent adaptive simulations.
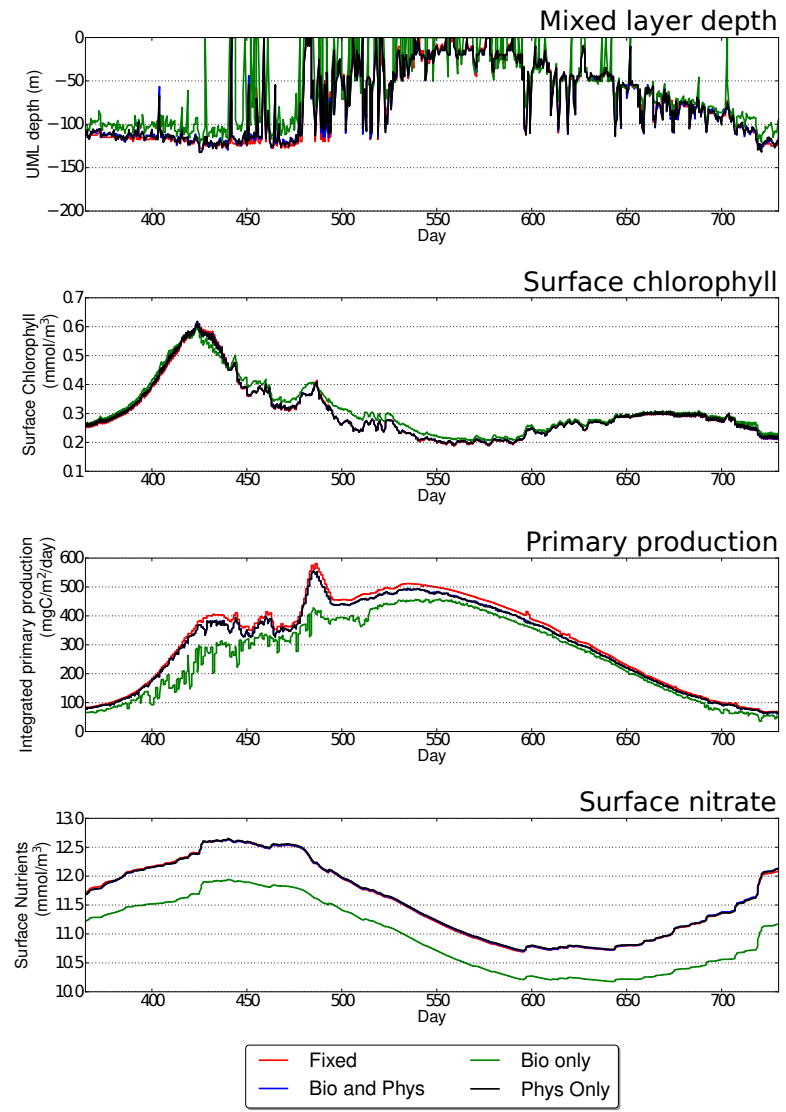

Fig. 12. Summary of results comparing different adaptivity metrics against measured data and the high-resolution $(2.5 \mathrm{~m})$ fixed-mesh simulation for Station Papa.

\subsection{Station Papa}

All adaptive simulations at Station Papa completed successfully and produced results that are a reasonable fit to measured data (Fig. 12). Using an adaptivity metric formed of physical or physical and biological quantities produces results that are not significantly different from that of the uniform $5 \mathrm{~m}$ resolution simulation (Table 5). All tracers give similar or better results than the $5 \mathrm{~m}$ fixed simulation, though given the lack of convergence shown in some of these quantities some caution must be used in interpreting these values. It is clear that using only biology or just PAR to form 
Table 6. The rms error, $\epsilon$, of adaptive mesh simulations compared to the simulation with $2.5 \mathrm{~m}$ vertical resolution at Bermuda. $\epsilon$ is shown for MLD, and the L2 2 norm of nutrient, primary productivity, chlorophyll and zooplankton. Note that the PAR and Bio only simulations failed and recorded no result. See Fig. 7 also.

\begin{tabular}{lrrrrrr}
\hline Res $(\mathrm{m})$ & $\epsilon_{\mathrm{MLD}}(\mathrm{m})$ & $\epsilon_{\mathrm{N}}\left(\mathrm{mmol} \mathrm{m}^{-3}\right)$ & $\epsilon_{\mathrm{P}}\left(\mathrm{mmol} \mathrm{m}^{-3}\right)$ & $\epsilon_{\mathrm{C}}\left(\mathrm{mmol} \mathrm{m}^{-3}\right)$ & $\epsilon_{\mathrm{Z}}\left(\mathrm{mmol} \mathrm{m}^{-3}\right)$ & No. elements (Mean, min, max) \\
\hline $5 \mathrm{~m}$ & 7.079 & 0.408 & 0.140 & 0.0129 & 0.0113 & 1200 \\
\hline Bio and Phys & 7.309 & 0.407 & 0.598 & 0.0084 & 0.0096 & $310.3,264,444$ \\
Phys Only & 8.432 & 0.408 & 0.615 & 0.0076 & 0.0082 & $228.3,174,436$ \\
\hline
\end{tabular}

Table 7. The rms error, $\epsilon$, of adaptive mesh simulations compared to the simulation with $2.5 \mathrm{~m}$ vertical resolution at Station India. $\epsilon$ is shown for MLD, and the L2 2 orm of nutrient, primary productivity, chlorophyll and zooplankton. See Fig. 8 also.

\begin{tabular}{lrrrrrr}
\hline Res $(\mathrm{m})$ & $\epsilon_{\mathrm{MLD}}(\mathrm{m})$ & $\epsilon_{\mathrm{N}}\left(\mathrm{mmol} \mathrm{m}^{-3}\right)$ & $\epsilon_{\mathrm{P}}\left(\mathrm{mmol} \mathrm{m}^{-3}\right)$ & $\epsilon_{\mathrm{C}}\left(\mathrm{mmol} \mathrm{m}^{-3}\right)$ & $\epsilon_{\mathrm{Z}}\left(\mathrm{mmol} \mathrm{m}^{-3}\right)$ & No. elements (Mean, min, max $)$ \\
\hline $5 \mathrm{~m}$ & 137.03 & 1.04 & 303.258 & 1.538 & 0.222 & 2400 \\
\hline Bio and Phys & 141.98 & 1.12 & 356.798 & 1.668 & 0.236 & $287.74,150.0,456.0$ \\
Bio Only & 112.72 & 1.12 & 324.777 & 1.052 & 0.097 & $220.63,120.0,360.0$ \\
Phys Only & 141.30 & 1.08 & 344.102 & 1.683 & 0.237 & $249.53,150.0,414.0$ \\
PAR & 131.84 & 1.17 & 677.312 & 1.920 & 0.245 & $120.00,120.0,121.0$ \\
\hline
\end{tabular}

the adaptivity metric is not adequate at this location as the values of the rms errors, $\epsilon$, for all tracers are substantially larger, apart from primary productivity, which has already been identified as potentially problematic in use as an assessment of performance.

\subsection{Bermuda}

Not all adaptive simulations were effective at Bermuda. Using either PAR or biology only to form the adaptivity metric results in simulations failing with a solver error soon after the first or second adapt. This is attributed to insufficient mesh resolution to ensure stability for the GLS turbulence parameterisations. Unlike Station Papa, the MLD is well below the photic zone in the initial stages of the simulation. However, both simulations using either physics only or biology and physics performed well. Both gave similar results, quantitatively (Table 6) and qualitatively (Fig. 13). The two adaptivity metrics also gave lower values of $\epsilon_{\mathrm{C}}$ and $\epsilon_{\mathrm{Z}}$, but, as with Station Papa, these values should interpreted with some caution.

\subsection{Station India}

All adaptive simulations produced good results at Station India when compared to the high-resolution fixed-mesh resolution regardless of adaptivity metric used (Table 7, Fig. 14). Minor differences in the timing of the spring bloom occurred with the biology-only adaptivity metric occurring some 25 days later than the fixed-mesh simulation. The biology-only adaptivity metric also showed an increase in the number of shoaling excursions in the spring. These did not occur when using other adaptivity metrics or in the fixed-mesh simulations. There are also minor differences in the magnitude of the integrated primary productivity, but these variations are much lower than those observed when changing resolution in the fixed-mesh simulations (Figs. 8).

\subsection{Summary of adaptive results}

Adaptive remeshing can clearly be successfully applied at the three ocean stations successfully using a variety of adaptivity metrics. Some adaptivity metric/station combinations perform better than others. As well as reproducing the surface values (i.e. at depth $z=0$ ) and the MLD the adaptive simulations also reproduce the vertical profiles of biological parameters (see Fig. 15 for chlorophyll and compare to Fig. 9).

The effect of adaptivity is clearly seen in the meshes produced by the simulations (Fig. 10). All stations show much higher resolution around the MLD, as expected, with decreased resolution when the MLD is deep (for example at Station India). The meshes contain far fewer elements than the high-resolution fixed-mesh simulations and are therefore more computationally efficient.

For all stations it is important to include velocity and density in the adaptivity metric (labelled as "physics" in all figures) and the inclusion of nutrients appears to be only of minor importance. However, using nutrients only or PAR can lead to a numerically unstable simulations and moreover, when the simulation is numerically stable, it gives much larger errors than the adaptivity metrics that include the physics. The simulated physics drives the biology and it is therefore crucial to include measures of the physical system in the adaptivity metric to correctly simulate the physical behaviour of the system. The physical measures used here are the vertical changes in velocity and density. However, turbulent kinetic energy, temperature or salinity may also be 

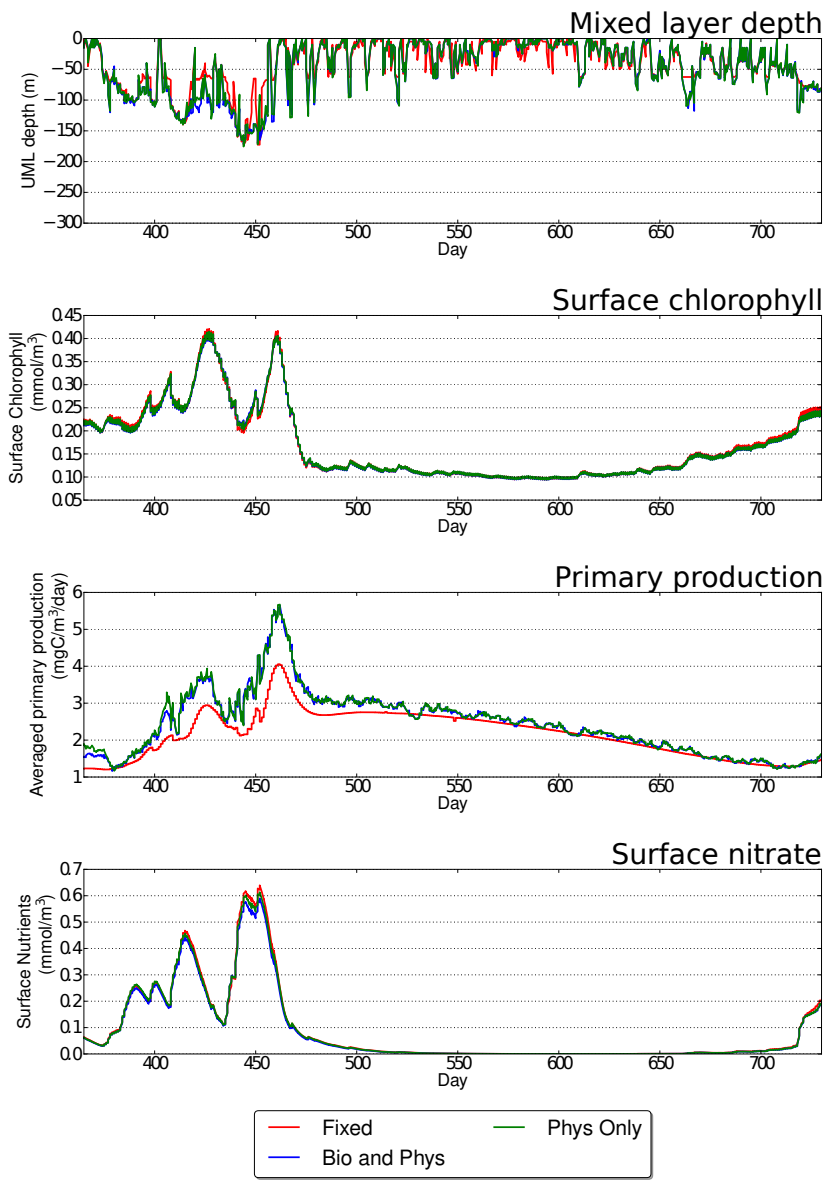

Fig. 13. Summary of results comparing different adaptivity metrics against measured data and the high-resolution $(2.5 \mathrm{~m})$ fixed-mesh simulation at Bermuda. Note that the simulations using PAR and biology only failed after only a few adapts.

sensible choices (Hill et al., 2012). Accounting for physical behaviour in the adaptivity metric appears to be sufficient for a successful simulation of biological behaviour. However, if the physical properties are well simulated then the biological processes do not necessarily also need including in the adaptivity metric in order to achieve a reasonable output.

\section{Specific adaptive examples}

One of the primary advantages of the approach outlined above is that the adaptivity metric used to calculate the mesh edge length can be composed of any simulated or diagnosed fields. We show the potential of that method here by simulating Bermuda with a adaptivity metric focusing on chlorophyll, and Station Papa concentrating on sinking detritus. These simulations show how the mesh is able to adapt to the particulars of the simulation, tracking transient behaviour but with a lower computational overhead than a high-resolution fixed-mesh simulation.
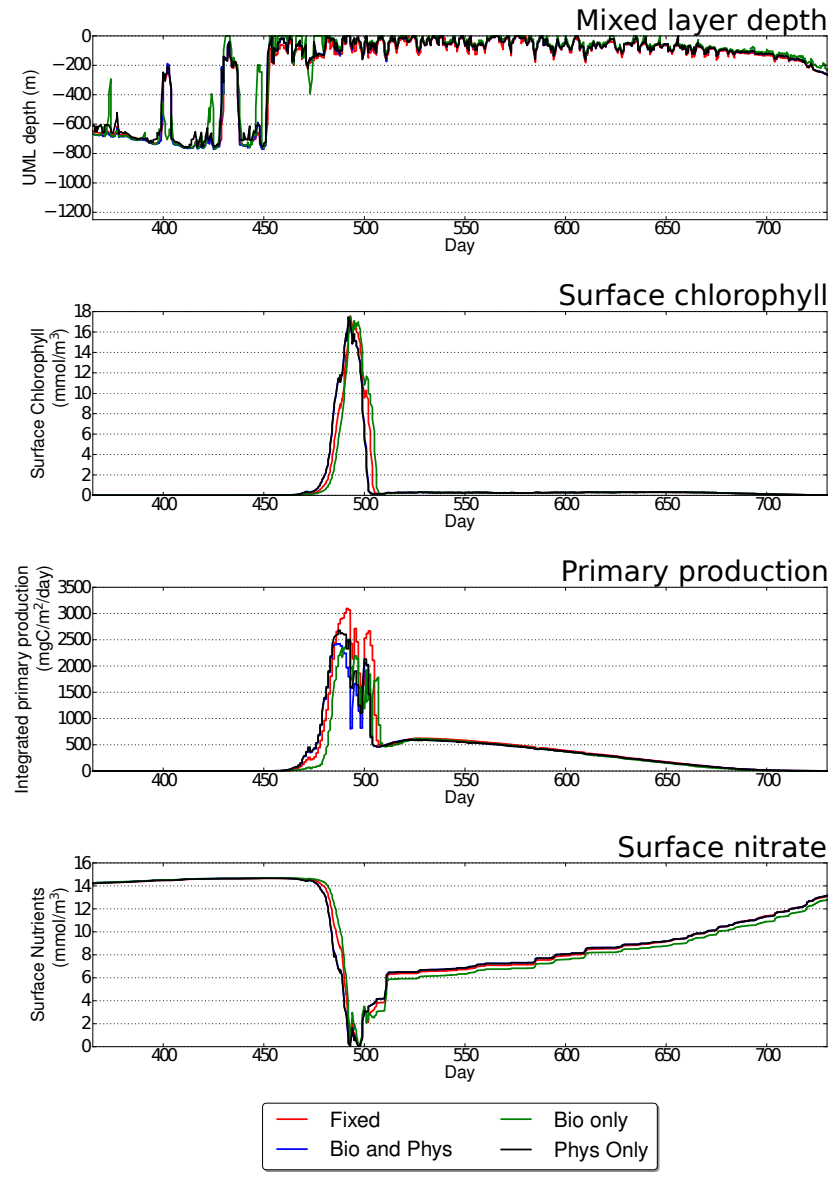

Fig. 14. Summary of results comparing different adaptivity metrics against measured data and the high-resolution $(2.5 \mathrm{~m})$ fixed-mesh simulation for Station India.

The first experiment attempts to track falling detritus in the Station Papa case. The detritus field is added to the adaptivity metric. The result, compared to the previous adaptive simulation using only nutrients, velocity and density in the adaptivity metric, shows a considerable change in the detritus concentration at depth (Fig. 16). In the original adaptive simulation, the detritus field is smoothed out at depths of over $300 \mathrm{~m}$ as the resolution here is relatively coarse. The effect of adding detritus to the adaptivity metric is as expected - the field maintains the sharp boundaries as it sinks, replicating the highest resolution fixed-mesh simulation. This is clearly seen in the resulting computational mesh (Fig. 17). In addition, we have carried out the same simulation based on a fixed mesh with decreasing resolution with increasing depth, as is typical in current ocean models. In this case we used the resolution change specified by the NEMO model (Madec, 2008) where resolution varies from $3 \mathrm{~m}$ at the surface to 100 by $1000 \mathrm{~m}$ depth. The results show increased diffusion of detritus by $100 \mathrm{~m}$ depth and by $700 \mathrm{~m}$ depth all detail in the detrital pulse is lost. 
A)

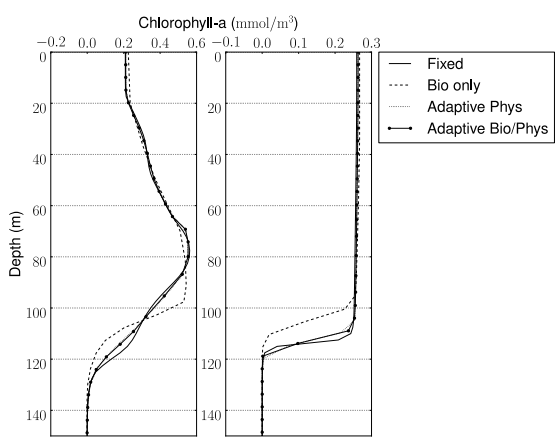

B)

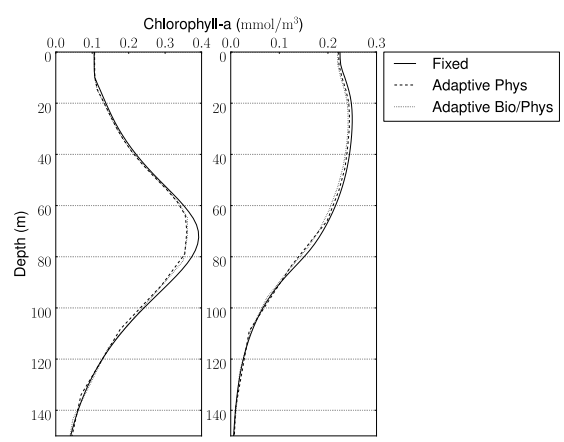

C)

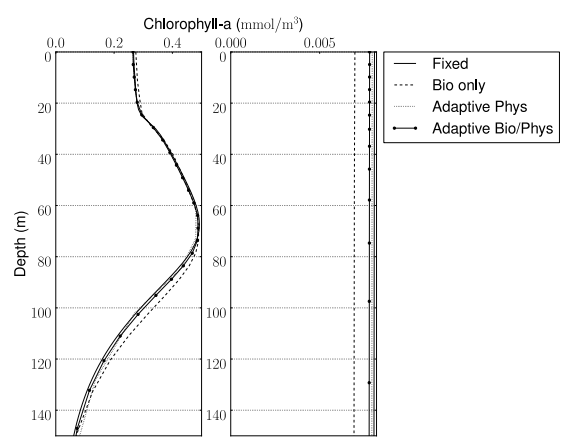

Fig. 15. Vertical profiles of chlorophyll at (A) Station Papa, (B) Bermuda and (C) Station India over the top $150 \mathrm{~m}, 150 \mathrm{~m}$ and $200 \mathrm{~m}$ respectively comparing the performance of different adaptivity metrics. The left-hand column shows the profile in summer (day 182) and the right-hand column shows the profile in winter (day 365 ). Note that all metrics, bar biology only, show similar results.

A similar result is seen at Bermuda where chlorophyll is added to the adaptivity metric. Here, we see that the subsurface chlorophyll maximum is maintained correctly (Fig. 18), whereas in the previous adaptive simulation the simulated value is lower around day 130 than for the fixed-mesh simulation. This is not the case when chlorophyll is added to the adaptivity metric. The simulation using a typical ocean model vertical resolution shows a lower chlorophyll peak (day 100) and a slightly reduced subsurface chlorophyll maximum throughout the summer compared to the highresolution simulation. The effect of adding chlorophyll to the adaptivity metric can be seen in the resulting mesh (Fig. 17).
For both simulations there is, of course, an increase in the number of elements used compared to the original adaptive simulations, but the average number of elements is still much lower than the high-resolution fixed-mesh simulation, and accordingly, the run times are much lower. The Bermuda simulation used an average of 437 elements (576 maximum, 301 minimum). Compared to a fixed mesh of uniform resolution $2.5 \mathrm{~m}$ ( 2400 elements) this is a fivefold reduction in elements on average. The simulation based on a typical ocean model vertical resolution uses 216 elements at the coast of reduced resolution of the chlorophyll maximum. Similarly, simulating detritus at Station Papa used an average of $726 \mathrm{el}-$ ements (507 minimum, 1120 maximum), compared to 2400 elements used in the $2.5 \mathrm{~m}$ fixed mesh simulation - a threefold reduction. The simulation using typical ocean model vertical resolution uses 216 elements (as at Bermuda) and is clearly incapable of accurately resolving sinking detritus.

\section{Conclusions}

This work has shown that the Fluidity model can successfully replicate expected biogeochemical behaviour at three key ocean stations. Both fixed and adaptive mesh simulations show very similar behaviour, but adaptive remeshing requires careful consideration of the adaptivity metric used. The physical quantities must be included in this adaptivity metric, and if the physical properties are well simulated then biological tracers do not necessarily need including in the adaptivity metric for reasonable output. This is consistent with a number of other studies where physical processes are the dominate control of ocean biogeochemical models (Berline et al., 2007). It is important to note that we have not shown an increase in model skill with either increasing resolution of when using adaptive meshes. We have, however, attempted to demonstrate numerical convergence of the biological model with increasing resolution.

It has been shown that a key component of successful adaptive simulation is to conservatively interpolate fields between the previous and new mesh following an adapt when using mesh hr-adaptivity. This is likely due to a change in mesh topology inducing spurious numerical diffusion and non-conservative methods are not suitable for biological tracers as conservation is important. The requirement of using conservative interpolation is likely down to topological changes to the mesh. Other adaptive mesh techniques such as mesh movement or $p$ adaptivity (where the order of the function space is altered) may not have such requirements. There is already work exploring the effectiveness of higher-order discretisations with biogeochemical models (Ueckermann and Lermusiaux, 2010). The work presented here will be a useful guide to future efforts in this area. In addition, we also found that for this quasi-1-D domain each column of vertices in the domain must be identical, such that there are no horizontal gradients. Fluidity currently uses a columnar mesh 

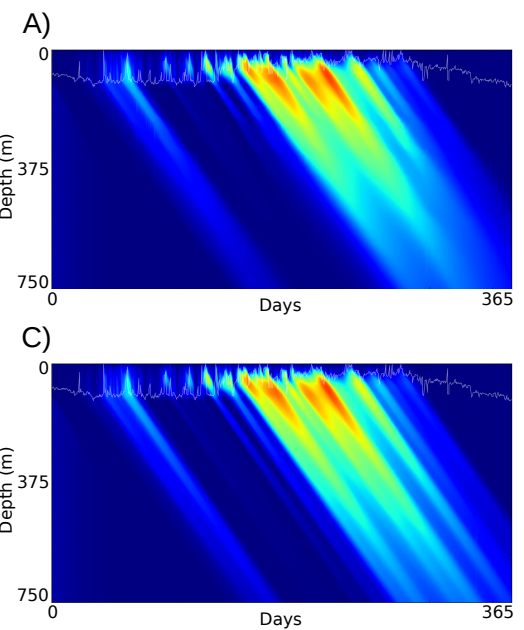

B)

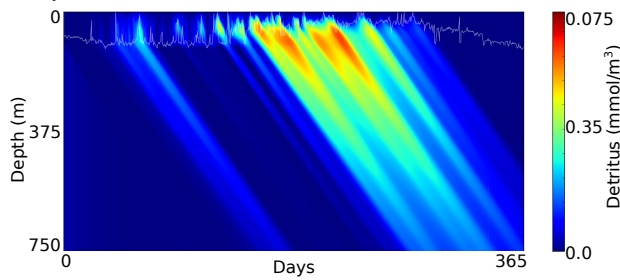

D)

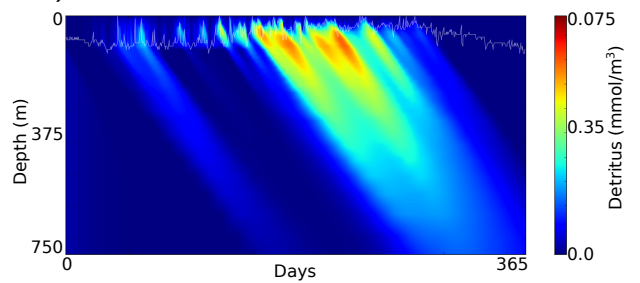

Fig. 16. Time-depth plot of detritus at Station Papa from the original adaptive mesh simulation (A), the adaptive run with detritus included in the metric (B), the high-resolution fixed-mesh simulation (C), and (D) the same simulation using decreasing resolution with increasing depth. Including detritus in the adaptive metric increases the performance of the model at tracking detritus to depth, resolving detail in the timing and level of detrital pulses. Note that (D) smooths those events into a single unresolved event by day 300.
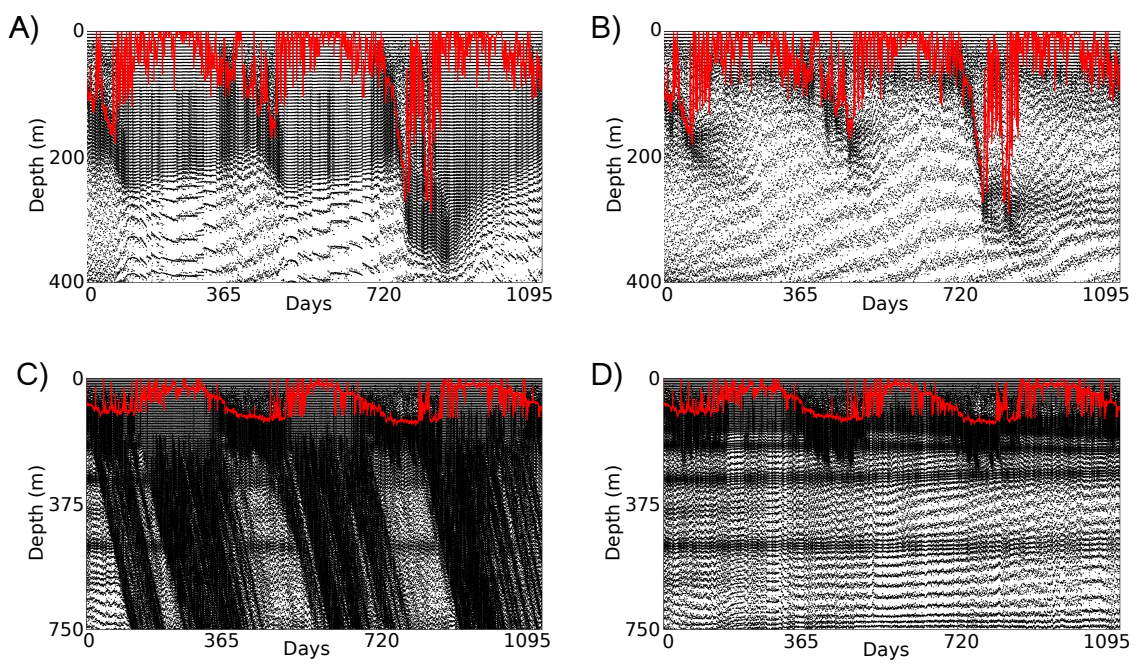

Fig. 17. Comparison of meshes produced by adaptive simulations. (A) Bermuda with the addition of chlorophyll to the adaptivity metric, (B) the original adaptive simulation at Bermuda using velocity, density and nutrients. Similarly, for Station Papa: (C) adaptive simulation with detritus included in the metric and (D) original adaptive simulation

in the vertical for large-scale ocean models (Piggott et al., 2008a) and is similar to the method used by Hofmeister et al. (2010). The work here is therefore compatible with how Fluidity will simulate global- or regional-scale models, using an unstructured horizontal mesh, with each vertical column then being extruded to the required depth. However, each column contains a different number of nodes in these cases which may induce additional vertical diffusivity due to horizontal gradients in the mesh. More work is required to ascertain if this effect is present in such simulations.
Ocean biogeochemical numerical models may require high numerical resolution in order to simulate measured data accurately (Oschlies, 2002). In addition, ocean biogeochemical models show sensitivity to a number of factors, such as light spectra (Kettle and Merchant, 2008) and surface fluxes (Friedrichs et al., 2006), but the main sensitivity is to the representation of the physics controlling the biology (e.g. Berline et al., 2007). A key part of this physical representation is the vertical advection and MLD behaviour. Regardless of whether a high horizontal resolution or adaptive horizontal meshes are used, the vertical resolution does not 

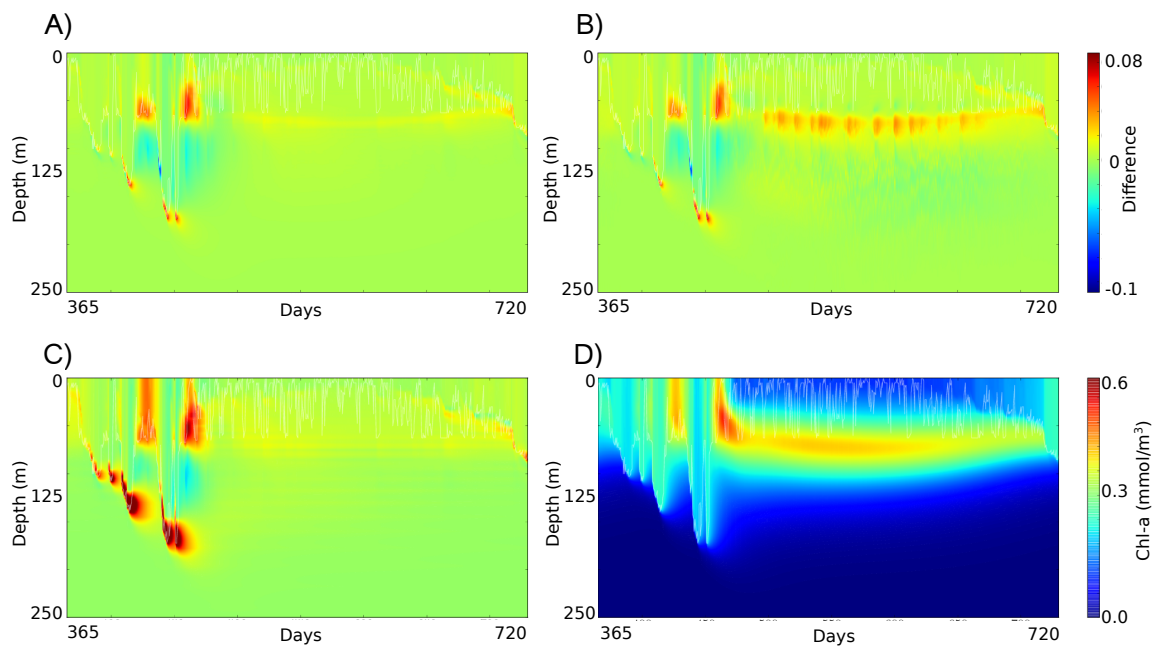

Fig. 18. Difference plots of chlorophyll at Bermuda comparing adaptive simulations (A and $\mathbf{B})$ and a fixed, but varying resolution mesh (C) to the highest resolution $(2.5 \mathrm{~m})$ uniform mesh simulation (D). A clear improvement can be made in the subsurface chlorophyll maximum representation by adding chlorophyll to the adaptivity metric. (A) The original adaptive mesh simulation shows larger discrepancies around the subsurface maximum (B). Using fixed variable resolution as in other ocean models shows clear discrepancies in the spring and throughout the summer (C).

need to be constant for each spatial location. Instead, as is shown here, the resolution only needs to be placed at key locations in the vertical. Enabling higher resolution around the MLD and within the upper layers of the ocean is sufficient for replicating high-resolution fixed-mesh simulations, hence adaptive remeshing may be advantageous. However, note that we have kept other parameters that may depend on resolution constant, including the biogeochemical model parameters. The turbulence parameterisation is not resolution dependant provided the model does not attempt to resolve the turbulent scales included in the parameterisation. This is the case here. For the stations simulated here, using the normal ocean model vertical resolution where resolution decreases with depth is sufficient to obtain a reasonable solution. However, mesh adaptivity also has another powerful feature: additions to the adaptivity metric of other fields allows for the tracking of transient features and of other fields, such as detritus or chlorophyll. This is a powerful tool in tracking features of interest whilst minimising the number of elements in the computational mesh.

In summary, adaptive remeshing shows good potential to reduce the number of required elements in the computational mesh whilst maintaining, or even increasing, the accuracy of vertical processes, but only where and when it is required. 


\section{Appendix A}

\section{Biology model}

As stated in the main body of the text, the biogeochemical model used in this report was based on that of Popova et al. (2006). Note, however, that the equations here are continuous for all depths, which was not true in Popova et al. (2006) where different source terms where used below the photic zone. The model parameters have also been fitted to match the data at all three test sites. The six components of the model are: nutrients (ammonium and nitrate), phytoplankton, chlorophyll, zooplankton and detritus.

\section{A1 Biological source terms}

The source terms for phytoplankton (P), chlorophyll (Chl), zooplankton (Z), nitrate (N), ammonium (A) and detritus (D) respectively are given by the following expressions:

$$
\begin{aligned}
S_{\mathrm{P}} & =\mathrm{P} J\left(Q_{\mathrm{N}}+Q_{\mathrm{A}}\right)-G_{\mathrm{P}}-\mathrm{De}_{\mathrm{P}}, \\
S_{\mathrm{Chl}} & =\left(R_{\mathrm{P}} \cdot J \cdot\left(Q_{\mathrm{N}}+\mathrm{Q}_{A}\right) \cdot \mathrm{P}\right. \\
& \left.\left.+\left(-G_{\mathrm{P}}-\mathrm{De}_{\mathrm{P}}\right)\right) \cdot \theta / \zeta\right), \\
S_{\mathrm{Z}} & =\delta \cdot\left(\beta_{\mathrm{P}} \cdot G_{\mathrm{P}}+\beta_{\mathrm{D}} \cdot G_{\mathrm{D}}\right)-\mathrm{De}_{\mathrm{Z}}, \\
S_{\mathrm{N}} & =-J \cdot \mathrm{P} \cdot Q_{\mathrm{N}}+\mathrm{De} \mathrm{e}_{\mathrm{A}}, \\
S_{\mathrm{A}} & =-J \cdot \mathrm{P} \cdot Q_{\mathrm{A}}+\mathrm{De} \mathrm{e}_{\mathrm{D}}+(1-\delta) \cdot\left(\beta_{\mathrm{P}} \cdot G_{\mathrm{P}}\right. \\
& \left.+\beta_{\mathrm{D}} \cdot G_{\mathrm{D}}\right)+(1-\gamma) \cdot \mathrm{De}_{\mathrm{Z}}-\mathrm{De}_{\mathrm{A}}, \\
S_{\mathrm{D}} & =-\mathrm{De} \mathrm{D}+\mathrm{De}+\gamma \cdot \mathrm{De}_{\mathrm{Z}} \\
& +\left(1-\beta_{\mathrm{P}}\right) \cdot G_{\mathrm{P}}-\beta_{\mathrm{D}} \cdot G_{\mathrm{D}} .
\end{aligned}
$$

The terms in these equations are given in Table A1.

Note that unlike the model of Popova et al. (2006) we use a continuous model, with no change of equations (bar one exception) above or below the photic zone. For our purposes, the photic zone is defined as $100 \mathrm{~m}$ water depth. First we calculate $\theta$ :

$\theta=\frac{\mathrm{Chl}}{\mathrm{P} \zeta}$.

However, at low light levels, Chl might be zero, therefore we take the limit that $\theta \rightarrow \zeta$ at low levels $\left(1 \times 10^{-7}\right)$ of chlorophyll or phytoplankton.

We then calculate $\alpha$ :

$\alpha=\alpha_{c} \theta$

using the PAR available at each vertex of the mesh the lightlimited phytoplankton growth rate, $J$, is then calculated:

$J=\frac{v \alpha I_{n}}{\sqrt{v^{2}+\alpha^{2}+I_{n}^{2}}}$.

This can be used to calculate the limiting factors on nitrate and ammonium:

$Q_{\mathrm{N}}=\frac{\mathrm{Nexp}^{-\Psi \mathrm{A}}}{K_{\mathrm{N}}+\mathrm{N}}$,
$Q_{\mathrm{A}}=\frac{\mathrm{A}}{K_{\mathrm{A}}+\mathrm{A}}$

From these the diagnostic field, primary production $\left(X_{\mathrm{P}}\right)$, can be calculated:

$X_{\mathrm{P}}=J\left(Q_{\mathrm{N}}+Q_{\mathrm{A}}\right) \mathrm{P}$.

The chlorophyll growth scaling factor is given by

$R_{\mathrm{P}}=Q_{\mathrm{N}} Q_{\mathrm{A}}\left(\frac{\theta_{m}}{\theta}\right)\left(\frac{v}{\sqrt{v^{2}+\alpha^{2}+I_{n}^{2}}}\right)$.

The zooplankton grazing terms are now calculated:

$G_{\mathrm{P}}=\frac{g p_{\mathrm{P}} \mathrm{P}^{2} Z}{\epsilon+\left(p_{\mathrm{P}} \mathrm{P}^{2}+p_{\mathrm{D}} \mathrm{D}^{2}\right)}$,

$G_{\mathrm{D}}=\frac{g p_{\mathrm{D}} \mathrm{D}^{2} \cdot \mathrm{Z}}{\epsilon+\left(p_{\mathrm{P}} \mathrm{P}^{2}+p_{\mathrm{D}} \mathrm{D}^{2}\right)}$.

Finally, the four death rates and re-mineralisation rates are calculated:

$\operatorname{De}_{\mathrm{P}}=\frac{\mu_{p} \mathrm{P}^{2}}{\mathrm{P}+k_{p}}+\lambda_{\text {bio }} \cdot \mathrm{P}$,
$\mathrm{De}_{\mathrm{Z}}=\frac{\mu_{z} \mathrm{Z}^{3}}{\mathrm{Z}+k_{z}}+\lambda_{\text {bio }} \cdot \mathrm{Z}$,

$\mathrm{De}_{\mathrm{D}}=\mu_{\mathrm{D}} \mathrm{D}+\lambda_{\text {bio }} \cdot \mathrm{P}+\lambda_{\text {bio }} \cdot \mathrm{Z}$

$\operatorname{De}_{\mathrm{A}}=\lambda_{\mathrm{A}} \mathrm{A}$ where $z<100$.

\section{A2 Photosynthetically active radiation (PAR)}

The photosynthetically active radiation, or PAR is calculated by

$\mathrm{PAR}=\left(A_{\text {water }}+A_{\mathrm{P}} \mathrm{P}\right) I$,

where $A_{\text {water }}$ and $A_{\mathrm{P}}$ are the absorption rates of photosynthetically active radiation by water and phytoplankton respectively.

\section{A3 Detritus sinking velocity}

Detritus is assumed to be denser than water and so sinks slowly through the water column. This is modelled by modifying the advecting velocity in the advection-diffusion equation for detritus by subtracting a sinking velocity $u_{\text {sink }}$ from the vertical component of the advecting velocity. 
Table A1. Symbols used to describe the six-component NPZD model. Typical values are provided for externally set parameters.

\begin{tabular}{|c|c|c|c|}
\hline Symbol & Meaning & Typical value & Equation \\
\hline$\alpha$ & initial slope of $P-I$ curve in $\left(\mathrm{W} \mathrm{m}^{-2}\right)^{-1} \mathrm{day}^{-1}$ & & Eq. (A8) \\
\hline$\alpha_{c}$ & Chl- $a$ specific initial slope of $P-I$ curve & $2 \mathrm{gC} \mathrm{gChl}^{-1}\left(\mathrm{~W} \mathrm{~m}^{-2}\right)^{-1} \mathrm{day}^{-1}$ & \\
\hline$\beta_{\mathrm{P}}, \beta_{\mathrm{D}}$ & assimilation coefficients of zooplankton & 0.75 & \\
\hline $\mathrm{De}_{\mathrm{D}}$ & rate of breakdown of detritus to ammonium & & Eq. (A18) \\
\hline $\operatorname{De}_{\mathrm{P}}$ & rate of phytoplankton natural mortality & & Eq. (A16) \\
\hline $\mathrm{De}_{\mathrm{Z}}$ & rate of zooplankton natural mortality & & Eq. (A17) \\
\hline $\mathrm{De}_{\mathrm{A}}$ & ammonium nitrification rate & & Eq. (A19) \\
\hline$\delta$ & excretion parameter & 0.7 & \\
\hline$\epsilon$ & grazing parameter relating capture of prey items to prey density & 0.4 & \\
\hline$G_{\mathrm{P}}$ & rate of zooplankton grazing on phytoplankton & & Eq. (A14) \\
\hline$G_{\mathrm{D}}$ & rate of zooplankton grazing on detritus & & Eq. (A15) \\
\hline$g$ & zooplankton maximum growth rate & 1.3 day $^{-1}$ & \\
\hline$\gamma$ & fraction of zooplankton mortality going to detritus & 0.5 & \\
\hline$I_{0}$ & $\begin{array}{l}\text { photosynthetically active radiation (PAR) immediately below } \\
\text { surface of water. Assumed to be } 0.43 \text { of the surface radiation }\end{array}$ & & \\
\hline$J$ & light-limited phytoplankton growth rate in day ${ }^{-1}$ & & Eq. (A9) \\
\hline$k_{\mathrm{A}}$ & half-saturation constant for ammonium uptake & $0.5 \mathrm{mmol} \mathrm{m}^{-3}$ & \\
\hline$k_{\mathrm{N}}$ & half-saturation constant for nitrate uptake & $0.5 \mathrm{mmol} \mathrm{m}^{-3}$ & \\
\hline$k_{\mathrm{P}}$ & half-saturation constant for phytoplankton mortality & $1 \mathrm{mmol} \mathrm{m}^{-3}$ & \\
\hline$k_{\mathrm{Z}}$ & half-saturation constant for zooplankton mortality & $3 \mathrm{mmol} \mathrm{m}^{-3}$ & \\
\hline$k_{w}$ & light attenuation due to water & $0.04 \mathrm{~m}^{-1}$ & \\
\hline$k_{c}$ & light attenuation due to phytoplankton & $0.03 \mathrm{~m}^{2} \mathrm{mmol}^{-1}$ & \\
\hline$\lambda_{\text {bio }}$ & rate of the phytoplankton and zooplankton transfer into detritus & 0.05 day $^{-1}$ & \\
\hline$\lambda_{\mathrm{A}}$ & nitrification rate & 0.03 day $^{-1}$ & \\
\hline$\mu_{\mathrm{P}}$ & phytoplankton mortality rate & 0.05 day $^{-1}$ & \\
\hline$\mu_{\mathrm{Z}}$ & zooplankton mortality rate & 0.2 day $^{-1}$ & \\
\hline$\mu_{\mathrm{D}}$ & detritus reference mineralisation rate & 0.05 day $^{-1}$ & \\
\hline$\Psi$ & strength of ammonium inhibition of nitrate uptake & $2.9\left(\mathrm{mmol} \mathrm{m}^{-3}\right)^{-1}$ & \\
\hline$p_{\mathrm{P}}$ & relative grazing preference for phytoplankton & 0.75 & \\
\hline$p_{\mathrm{D}}$ & relative grazing preference for detritus & 0.25 & \\
\hline$Q_{\mathrm{N}}$ & non-dimensional nitrate limiting factor & & Eq. (A10) \\
\hline$Q_{\mathrm{A}}$ & non-dimensional ammonium limiting factor & & Eq. (A11) \\
\hline$R_{\mathrm{P}}$ & Chl growth scaling factor & & Eq. (A13) \\
\hline$v$ & Maximum phytoplankton growth rate & 1 day $^{-1}$ & \\
\hline$w_{g}$ & detritus sinking velocity & $10 \mathrm{~m} \mathrm{day}^{-1}$ & \\
\hline$z$ & depth & & \\
\hline$\theta$ & 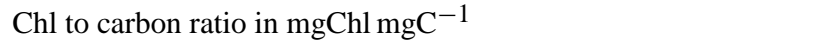 & & \\
\hline$\theta_{m}$ & maximum Chl to carbon ratio & $0.05 \mathrm{mg} \mathrm{Chl} \mathrm{mgC}-1$ & \\
\hline$\zeta$ & conversion factor from $\mathrm{gC}$ to $\mathrm{mmolN}$ based on $\mathrm{C}: \mathrm{N}$ ratio of 6.5 & $0.0128 \mathrm{mmolN} \mathrm{ngC}^{-1}$ & \\
\hline
\end{tabular}


Acknowledgements. All authors were funded by NERC under grants NE/F00270X/1 and NE/F004184/1. The authors would like to acknowledge the use of the Imperial College London HPC service to perform some of the simulations presented here. The authors would like to thank the two anonymous reviewers for their helpful comments and suggestions.

Edited by: E. J. M. Delhez

\section{References}

Anderson, T. R. and Pondaven, P.: Non-redfield carbon and nitrogen cycling in the Sargasso Sea: pelagic imbalances and export flux, Deep Sea Res.-Pt. I, 50, 573-591, doi:10.1016/S09670637(03)00034-7, 2003.

Berline, L., Brankart, J.-M., Brasseur, P., Ourmières, Y., and Verron, J.: Improving the physics of a coupled physicalbiogeochemical model of the North Atlantic through data assimilation: Impact on the ecosystem, J. Mar. Syst., 64, 153-172, doi:10.1016/j.jmarsys.2006.03.007, 2007.

Burchard, H. and Beckers, J.-M.: Non-uniform adaptive vertical grids in one-dimensional numerical ocean models, Ocean Model., 6, 51-81, doi:10.1016/S1463-5003(02)00060-4, 2004.

Burchard, H. and Bolding, K.: Comparative Analysis of Four Second-Moment Turbulence Closure Models for the Oceanic Mixed Layer, J. Phys. Oceanogr., 31, 1943-1968, doi:10.1175/1520-0485(2001)031<1943:CAOFSM>2.0.CO;2, 2001.

Denman, K. and Miyake, M.: Upper Layer Modification at Ocean Station Papa: Observations and Simulation, J. Phys. Oceanogr., 3, 185-196, 1973.

Farrell, P., Piggott, M., Pain, C., Gorman, G., and Wilson, C.: Conservative interpolation between unstructured meshes via supermesh construction, Comput. Method. Appl. M., 198, 2632-2642, doi:10.1016/j.cma.2009.03.004, 2009.

Ford, R., Pain, C. C., Piggott, M. D., Goddard, A. J. H., de Oliveira, C. R. E., and Umpleby, A. P.: A Nonhydrostatic Finite-Element Model for Three-Dimensional Stratified Oceanic Flows. Part I: Model Formulation, Mon. Weather Rev., 132, 2816-2831, doi:10.1175/MWR2824.1, 2004.

Friedrichs, M. A., Hood, R. R., and Wiggert, J. D.: Ecosystem model complexity versus physical forcing: Quantification of their relative impact with assimilated Arabian Sea data, Deep Sea Res.-Pt. II, 53, 576-600, doi:10.1016/j.dsr2.2006.01.026, 2006.

Gaspar, P., Grégoris, Y., Lefevre, J.-M., and Goris, Y. G. R. I.: A Simple Eddy Kinetic Energy Model for Simulations of the Oceanic Vertical Mixing: Tests at Station Papa and Long-Term Upper Ocean Study Site, J. Geophys. Res., 95, 16179-16193, doi:10.1029/JC095iC09p16179, 1990.

Hanert, E., Deleersnijder, E., and Legat, V.: An adaptive finite element water column model using the Mellor-Yamada level 2.5 turbulence closure scheme, Ocean Model., 12, 205-223, doi:10.1016/j.ocemod.2005.05.003, 2006.

Hiester, H., Piggott, M., and Allison, P.: The impact of mesh adaptivity on the gravity current front speed in a two-dimensional lock-exchange, Ocean Model., 38, 1-21, doi:10.1016/j.ocemod.2011.01.003, 2011.

Hill, J., Piggott, M., Ham, D., Popova, E., and Srokosz, M.: On the performance of a generic length scale turbulence model within an adaptive finite element ocean model, Ocean Model., 56, 1-15, doi:10.1016/j.ocemod.2012.07.003, 2012.

Hofmeister, R., Burchard, H., and Beckers, J.-M.: Non-uniform adaptive vertical grids for 3D numerical ocean models, Ocean Model., 33, 70-86, doi:10.1016/j.ocemod.2009.12.003, 2010.

Ji, R., Davis, C., Chen, C., and Beardsley, R.: Influence of local and external processes on the annual nitrogen cycle and primary productivity on Georges Bank: A 3-D biological-physical modeling study, J. Mar. Syst., 73, 31-47, doi:10.1016/j.jmarsys.2007.08.002, 2008.

Kettle, H. and Merchant, C. J.: Modeling ocean primary production: Sensitivity to spectral resolution of attenuation and absorption of light, Prog. Oceanogr., 78, 135-146, doi:10.1016/j.pocean.2008.04.002, 2008.

Khangaonkar, T., Sackmann, B., Long, W., Mohamedali, T., and Roberts, M.: Simulation of annual biogeochemical cycles of nutrient balance, phytoplankton bloom(s), and DO in Puget Sound using an unstructured grid model, Ocean Dynam., 62, 13531379, doi:10.1007/s10236-012-0562-4, 2012.

Kleypas, J. A. and Doney, S. C.: Nutrients, Chlorophyll, Primary Production and related Biogeochemical Properties in the Ocean Mixed Layer, Tech. Rep. TN-447+STR, NCAR, available at: http://rda.ucar.edu/datasets/ds259.0/ (last access: 8 May 2014), 2001.

Large, W. G. and Yeager, S. G.: Diurnal to decadal global forcing for ocean and sea-ice models: The data sets and flux climatologies, Tech. Rep. Technical Report TN-460+STR, NCAR, 2004.

Lévy, M., Iovino, D., Resplandy, L., Klein, P., Madec, G., Tréguier, A.-M., Masson, S., and Takahashi, K.: Largescale impacts of submesoscale dynamics on phytoplankton: Local and remote effects, Ocean Model., 43-44, 77-93, doi:10.1016/j.ocemod.2011.12.003, 2012.

Loseille, A. and Alauzet, F.: Continuous Mesh Framework Part II: Validations and Applications, SIAM J. Numer. Anal., 49, 61-86, doi:10.1137/10078654X, 2011.

Luo, L., Wang, J., Schwab, D. J., Vanderploeg, H., Leshkevich, G., Bai, X., Hu, H., and Wang, D.: Simulating the 1998 spring bloom in Lake Michigan using a coupled physicalbiological model, J. Geophys. Res.-Oceans, 117, C10011, doi:10.1029/2012JC008216, 2012.

Madec, G.: NEMO ocean engine, Note du Pole de modélisation, Institut Pierre-Simon Laplace (IPSL), France, No. 27 ISSN No 1288-1619, 2008.

McGillicuddy, D. J., Anderson, L. A., Doney, S. C., and Maltrud, M. E.: Eddy-driven sources and sinks of nutrients in the upper ocean: Results from a $0.1^{\circ}$ resolution model of the North Atlantic, Global Biogeochem. Cy., 17, 1035, doi:10.1029/2002GB001987, 2003.

Menendez, A. N., Badano, N. D., Lopolito, M. F., and Re, M.: Water quality assessment for a coastal zone through numerical modeling, J. Appl. Water Eng. Res., 1, 8-16, doi:10.1080/23249676.2013.827892, 2013.

Oschlies, A.: Can eddies make ocean deserts bloom?, Global Biogeochem. Cy., 16, 1106, doi:10.1029/2001GB001830, 2002.

Oschlies, A. and Garçon, V.: An eddy-permitting coupled physicalbiological model of the North Atlantic: 1 . Sensitivity to advection numerics and mixed layer physics, Global Biogeochem. Cy., 13, 135-160, doi:10.1029/98GB02811, 1999. 
Pain, C. C., Umpleby, A. P., de Oliveira, C. R. E., and Goddard, A. J. H.: Tetrahedral mesh optimisation and adaptivity for steadystate and transient finite element calculations, Comput. Method Appl. M., 190, 3771-3796, doi:10.1016/S0045-7825(00)002942, 2001.

Pain, C., Piggott, M., Goddard, A., Fang, F., Gorman, G., Marshall, D., Eaton, M., Power, P., and de Oliveira, C.: Threedimensional unstructured mesh ocean modelling, Ocean Model., 10, 5-33, doi:10.1016/j.ocemod.2004.07.005, the Second International Workshop on Unstructured Mesh Numerical Modelling of Coastal, Shelf and Ocean Flows, 2005.

Pichelina, É., Fortina, M., and Boivinb, S.: Étude numérique d'estimations d'erreur a posteriori, Revue Européenne des Éléments, 9, 467-486, 2000.

Piggott, M. D., Pain, C. C., Gorman, G. J., Power, P. W., and Goddard, A. J. H.: h, r, and hr adaptivity with applications in numerical ocean modelling, Ocean Model., 10, 95-113, doi:10.1016/j.ocemod.2004.07.007, 2005.

Piggott, M., Pain, C., Gorman, G., Marshall, D., Marshall, D. P., and Killworth, P. D.: Unstructured adaptive meshes for ocean modeling, in: Ocean Modeling in an Eddying Regime. Geophysical Monograph Series, 177, doi:10.1029/177GM22, 2008a.

Piggott, M. D., Gorman, G. J., Pain, C. C., Allison, P. A., Candy, A. S., Martin, B. T., and Wells, M. R.: A new computational framework for multi-scale ocean modelling based on adapting unstructured meshes, Int. J. Numer. Meth. Fl., 56, 1003-1015, doi:10.1002/fld.1663, 2008b.

Piggott, M. D., Farrell, P. E., Wilson, C. R., Gorman, G. J., and Pain, C. C.: Anisotropic mesh adaptivity for multi-scale ocean modelling, Philos. T. Roy. Soc. A, 367, 4591-4611, doi:10.1098/rsta.2009.0155, 2009.
Popova, E. E., Coward, A. C., Nurser, G. A., de Cuevas, B., Fasham, M. J. R., and Anderson, T. R.: Mechanisms controlling primary and new production in a global ecosystem model - Part I: Validation of the biological simulation, Ocean Sci., 2, 249-266, doi:10.5194/os-2-249-2006, 2006.

Ueckermann, M. P. and Lermusiaux, P. F. J.: High-order schemes for 2D unsteady biogeochemical ocean models, Ocean Dynam., 60, 1415-1445, doi:10.1007/s10236-010-0351-x, 2010.

Umlauf, L. and Burchard, H.: A generic length-scale equation for geophysical turbulence models, J. Mar. Res., 61, 235-265, doi:10.1357/002224003322005087, 2003.

Uppala, S. M., Kållberg, P. W., Simmons, A. J., Andrae, U., Bechtold, V. D. C., Fiorino, M., Gibson, J. K., Haseler, J., Hernandez, A., Kelly, G. A., Li, X., Onogi, K., Saarinen, S., Sokka, N., Allan, R. P., Andersson, E., Arpe, K., Balmaseda, M. A., Beljaars, A. C. M., Berg, L. V. D., Bidlot, J., Bormann, N., Caires, S., Chevallier, F., Dethof, A., Dragosavac, M., Fisher, M., Fuentes, M., Hagemann, S., Hólm, E., Hoskins, B. J., Isaksen, L., Janssen, P. A. E. M., Jenne, R., Mcnally, A. P., Mahfouf, J.-F., Morcrette, J.-J., Rayner, N. A., Saunders, R. W., Simon, P., Sterl, A., Trenberth, K. E., Untch, A., Vasiljevic, D., Viterbo, P., and Woollen, J.: The ERA-40 re-analysis, Q. J. Roy. Meteorol. Soc., 131, 2961-3012, doi:10.1256/qj.04.176, 2005.

Weber, L., Völker, C., Oschlies, A., and Burchard, H.: Iron profiles and speciation of the upper water column at the Bermuda Atlantic Time-series Study site: a model based sensitivity study, Biogeosciences, 4, 689-706, doi:10.5194/bg-4-689-2007, 2007. 